TRANSACTIONS OF THE

AMERICAN MATHEMATICAL SOCIETY

Volume 362, Number 9, September 2010, Pages 4955-4981

S 0002-9947(10)05089-0

Article electronically published on April 21, 2010

\title{
TIME-FREQUENCY REPRESENTATIONS OF WIGNER TYPE AND PSEUDO-DIFFERENTIAL OPERATORS
}

\author{
P. BOGGIATTO, G. DE DONNO, AND A. OLIARO
}

\begin{abstract}
We introduce a $\tau$-dependent Wigner representation, $\mathrm{Wig}_{\tau}, \tau \in$ $[0,1]$, which permits us to define a general theory connecting time-frequency representations on one side and pseudo-differential operators on the other. The scheme includes various types of time-frequency representations, among the others the classical Wigner and Rihaczek representations and the most common classes of pseudo-differential operators. We show further that the integral over $\tau$ of $\mathrm{Wig}_{\tau}$ yields a new representation $Q$ possessing features in signal analysis which considerably improve those of the Wigner representation, especially for what concerns the so-called "ghost frequencies". The relations of all these representations with respect to the generalized spectrogram and the Cohen class are then studied. Furthermore, a characterization of the $L^{p}$-boundedness of both $\tau$-pseudo-differential operators and $\tau$-Wigner representations are obtained.
\end{abstract}

\section{INTRODUCTION}

One basic drawback of the classical Fourier transform

$$
\mathcal{F} f(\omega)=\hat{f}(\omega)=\int_{\mathbb{R}^{d}} e^{-2 \pi i t \omega} f(t) d t, \quad t, \omega \in \mathbb{R}^{d},
$$

in its applications to signal analysis is that information concerning the time at which frequencies appear in a signal $f(t)$ are hidden in its complex phase. As a consequence one of the main aims of the time-frequency analysis over the last 50 years has been to define suitable "2-variable" modifications of the Fourier transform in such a way that information about both time and frequency content of the signal are made explicit. They are functions (or distributions) $\Psi(f)(x, \omega)$, both of the time variables $x \in \mathbb{R}^{d}$ and the frequency variables $\omega \in \mathbb{R}^{d}$, depending in a quadratic way on the signal $f$. Whereas a single point value $\Psi(f)(x, \omega)$ does not have a physical interpretation, the amplitude of $\Psi(f)$ around a point $(x, \omega)$ indicates, roughly speaking, the magnitude of the frequencies $\omega$ present in the signal $f$ around the time $x$. Due to its physical interpretation as energy distribution of the signal in the time-frequency space $\mathbb{R}_{x}^{d} \times \mathbb{R}_{\omega}^{d}$, we shall indifferently use in this context the term "representation" or (quadratic) "form" for $\Psi$. This suggests furthermore that it is desirable for a "suitable" $\Psi(f)$ to satisfy some typical conditions of density functions analogous to those in statistics and probability. It turns out, however, that, different to what happens in statistics and probability theory, the situation in time-frequency analysis is more similar to that of quantum mechanics where, as a consequence

Received by the editors April 3, 2008 and, in revised form, April 17, 2009.

2010 Mathematics Subject Classification. Primary 47G30; Secondary 35S05, 42B10, 44A35, $47 \mathrm{~B} 38$.

(C)2010 American Mathematical Society 4955

Reverts to public domain 28 years from publication 
of the uncertainty principle, the natural requirements on the distribution density of the energy are actually incompatible with one another and can therefore be satisfied only with a certain degree of approximation. For this reason many different representations have been defined in literature, which, according to the features they possess, can be more suitable for one application than another. For details on this subject see [6], 7], 8], 9, [12, [13.

In this paper we introduce new types of representations $W i g_{\tau}$, a modification of the Wigner representation depending on a parameter $\tau \in[0,1]$.

These representations turn out to play a crucial role both within the theory of time-frequency representations and for what concerns its connections with the theory of pseudo-differential operators.

We shall actually show that the Rihaczek, the Wigner and the "adjoint"-Rihaczek representations are obtained as particular cases of the $W i g_{\tau}$ representations in correspondence to the values $\tau=0, \tau=1 / 2, \tau=1$, respectively. We then consider the integral of the representation-valued function $\tau \in[0,1] \longmapsto W i g_{\tau}$ over the interval $[0,1]$ defining a new form $Q=\int_{[0,1]} W i g_{\tau} d \tau$. The aim of this definition is that the form $Q$ will turn out to be a considerably better representation than every single $W i g_{\tau}$, in particular better than the Wigner distribution. More precisely, $Q$ enjoys all the "good" features of the Wigner distribution, but, in comparison with it, it practically eliminates the presence of the so-called "ghost frequencies". We shall illustrate how this happens in section 3 and give a precise mathematical framework in section 4 .

We then proceed to give a detailed study of $W i g_{\tau}$ representations and their integral $Q$, namely we prove that they belong to the Cohen class but not to the generalized spectrogram (with the exception of the extreme cases $W i g_{\tau}, \tau=0$, $\tau=1$ ) and that they satisfy the marginal distribution conditions and enjoy the support property, therefore being valuable candidates for a time-frequency analysis of signals. Furthermore, the integral representation $Q$, as the Wigner representation, is real for every signal $f$.

The other topic where the $W i g_{\tau}$ representations appear to be of considerable interest are the connections between time-frequency distributions and pseudo-differential operators.

Representations are deeply related to pseudo-differential operators. In particular, under suitable conditions, a one-to-one correspondence can be established and it can be proved that boundedness of representations is equivalent to that of the corresponding operators; see [2], [3, [4].

We shall prove that a new remarkable couple "representation-operator" fits into this scheme. Namely, the $\tau$-Wigner representations exactly correspond to "pseudodifferential operators with $\tau$-symbol", which are widely use in literature; see [16]. This gives rise to a somewhat complete scheme summarized in (2.11).

We shall then characterize $L^{p}$-boundedness both for $\tau$ - representations and $\tau$ pseudo-differential operators.

\section{A GeNERAL SCHEME OF REPRESENTATIONS} AND PSEUDO-DIFFERENTIAL OPERATORS

The classical "Wigner" representation is the form

$$
W i g(f)(x, \omega)=\int_{\mathbb{R}^{d}} e^{-2 \pi i t \omega} f(x+t / 2) \overline{f(x-t / 2)} d t
$$


defined by E. Wigner in the context of quantum mechanics, where, in the search for ideal position-momentum distributions, questions arise which are analogous to those of time-frequency analysis (see [20]). It is not positive, but it enjoys the support property and satisfies the marginals (see [6], [10, [12]).

With abuse of notation 11 we shall use the same name for the corresponding polarized sesquilinear form

$$
W i g(f, g)(x, \omega)=\int_{\mathbb{R}^{d}} e^{-2 \pi i t \omega} f(x+t / 2) \overline{g(x-t / 2)} d t
$$

Based on the Wigner representation is the so-called Cohen class, i.e. the set of representations of the form

$$
Q(f, g)=W i g(f, g) * \sigma
$$

with $\sigma \in \mathcal{S}^{\prime}\left(\mathbb{R}^{2 d}\right)$. This very large and interesting class includes most of the more commonly used representations of time-frequency analysis; see [5], [6], [10].

In [2], 3] a particular subset of the Cohen class named generalized spectrogram was defined. They are representations $S p_{\phi, \psi}$ depending on two windows $\phi, \psi$ which, when suitably "moved", can make the corresponding spectrograms define a link between some well-known representations, namely classical spectrograms and Rihaczek and conjugate-Rihaczek representations. We now briefly summarize these ideas (for details see [2] and [3]), as in this paper we shall propose a similar "path" of representations also laying in the Cohen class but "outside" the generalized spectrogram class.

The generalized spectrogram is the sesquilinear form

$$
S p_{\phi, \psi}(f, g)(x, \omega)=V_{\phi} f(x, \omega) \overline{V_{\psi} g(x, \omega)},
$$

where $V_{\phi} f(x, \omega)=\int_{\mathbb{R}^{d}} f(t) e^{-2 \pi i t \omega} \overline{\phi(t-x)} d t$ is the Gabor transform of $f$ with window $\phi$ (also known as a Short-time Fourier transform or Windowed Fourier transform).

The fact that the generalized spectrogram is a subclass of the Cohen class follows from the convolution formula (see [2])

$$
V_{\phi} f \overline{V_{\psi} g}=W i g(\tilde{\psi}, \tilde{\phi}) * W i g(f, g)
$$

with $\tilde{F}(x)=F(-x)$, which expresses the generalized spectrogram as a convolution of Wigner transforms.

In [3] it is further proved that the Wigner representation is not a generalized spectrogram, therefore showing that the latter is a proper subclass of the Cohen class.

On the contrary the classical spectrogram with window $\phi$ is trivially obtained by taking equal windows $\phi=\psi$ in (2.3) and is the only positive representation among the generalized spectrograms.

The other very basic representation which is proved in [3] to be in the generalized spectrogram is the Rihaczek form

$$
R(f, g)(x, \omega)=e^{-2 \pi i x \omega} f(x) \overline{\hat{g}(\omega)}
$$

It is proved more precisely that the Rihaczek representation coincides with the particular "limit" case of spectrogram corresponding to windows $\phi=\delta, \psi=1$

\footnotetext{
${ }^{1}$ We shall adopt this notation for all representations.
} 
(where $\delta$ is the Dirac point distribution). Symmetrically if $\phi=1, \psi=\delta$ are taken as windows, we obtain the "conjugate"-Rihaczek representation:

$$
R^{*}(f, g)(x, \omega)=\overline{R(g, f)(x, \omega)}=e^{2 \pi i x \omega} \overline{g(x)} \hat{f}(\omega) .
$$

More explicitly we have

$$
\begin{aligned}
& R(f, g)=S p_{\delta, 1}(f, g)=W i g(1, \delta) * W i g(f, g)=e^{-4 \pi i x \omega} * W i g(f, g)(x, \omega), \\
& R^{*}(f, g)=S p_{1, \delta}(f, g)=W i g(\delta, 1) * W i g(f, g)=e^{4 \pi i x \omega} * W i g(f, g)(x, \omega) .
\end{aligned}
$$

An explicit "path" of representations connecting spectrogram, Rihaczek and conjugate Rihaczek representations is for example $S p_{\phi_{\lambda}, \hat{\phi}_{\lambda}}, \quad \lambda \in[0, \infty]$, where the $L^{1}$-normalized gaussians $\phi_{\lambda}(x)=\lambda^{d / 2} e^{-\pi \lambda x^{2}}$ and their Fourier transforms are used as windows (with the convention $\phi_{\infty}=\delta$ ).

We next show that a similar situation, i.e. a link between Rihaczek and conjugate Rihaczek representations, can also be defined outside the generalized spectrogram class and that it is actually constituted by a path of representations depending on a parameter $\tau \in[0,1]$, which we shall call $\mathrm{Wig}_{\tau}$, having as a "middle point" the Wigner representation; see Figure 1. The motivation of the definition of these $\tau$-dependent representations is not merely the fact that the path they describe includes some very well-known representations; two other facts are even more remarkable: their integral over the parameter $\tau$ will yield an "integral" representation $Q$ (see Definition 2.3), which, as we shall see in section 3, shows considerably better behavior than the Wigner representation, especially with respect to the problem of the so-called "ghost frequencies". Furthermore the $W i g_{\tau}$ forms exactly correspond to the well-known $\tau$-pseudo-differential calculus in a general form-operator correspondence (see Propositions 2.4 and 2.6), therefore allowing a nice symmetric picture of operators and forms in a considerably wide variety of significant cases.

More precisely we define the $\tau$-Wigner representation as follows.

Definition 2.1. For $\tau \in[0,1], f, g \in \mathcal{S}\left(\mathbb{R}^{d}\right)$ let

$$
W i g_{\tau}(f, g)(x, \omega)=\int_{\mathbb{R}^{d}} e^{-2 \pi i t \omega} f(x+\tau t) \overline{g(x-(1-\tau) t)} d t .
$$

Here we summarize the main features of the $\tau$-Wigner representations that shall be proved in section 5 .

\section{Proposition 2.2.}

- $W_{\tau}$ is in the Cohen class for every $\tau \in[0,1]$,

$-W i g_{0}=R$ (Rihaczek representation),

- Wig $_{1 / 2}=W i g$ (Wigner representation),

$-W i g_{1}=R^{*} \quad$ (conjugate Rihaczek representation),

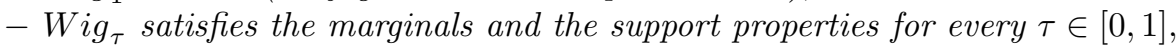

- Wig ${ }_{1-\tau}(f, g)=\overline{W i g_{\tau}(g, f)}$.

From the last formula we have

$$
\begin{aligned}
& W i g_{\tau}(f)+W i g_{1-\tau}(f)=2 \Re\left(W i g_{\tau}(f)\right), \\
& W i g_{\tau}(f)-W i g_{1-\tau}(f)=2 i \Im\left(W i g_{\tau}(f)\right) .
\end{aligned}
$$

The first of these two formulas suggests that even if generally $W i g_{\tau}(f)$ is not real, integration with respect to $\tau$ on any interval with midpoint $1 / 2$ yields a representation which is real for every signal $f$ and in some sense represents a mean of the 
single $\tau$-Wigner representations. We are then led in a somewhat natural way to the following:

Definition 2.3. For $\mathrm{Wig}_{\tau}$ as in Definition 2.1 we define the new representation:

$$
Q(f, g)(x, \omega)=\int_{[0,1]} W_{\tau} g_{\tau}(f, g)(x, \omega) d \tau .
$$

As mentioned above the form $Q$ has considerable advantages with respect to $W i g_{\tau}$, Wigner representation included. We shall show that $Q$ is a real representation belonging to the Cohen class and satisfies marginals and support conditions. Section 3 is dedicated to comparing its features with those of $W i g_{\tau}$, therefore motivating its introduction. In Section 3 the basic properties of $Q$ and $W i g_{\tau}$ are studied.

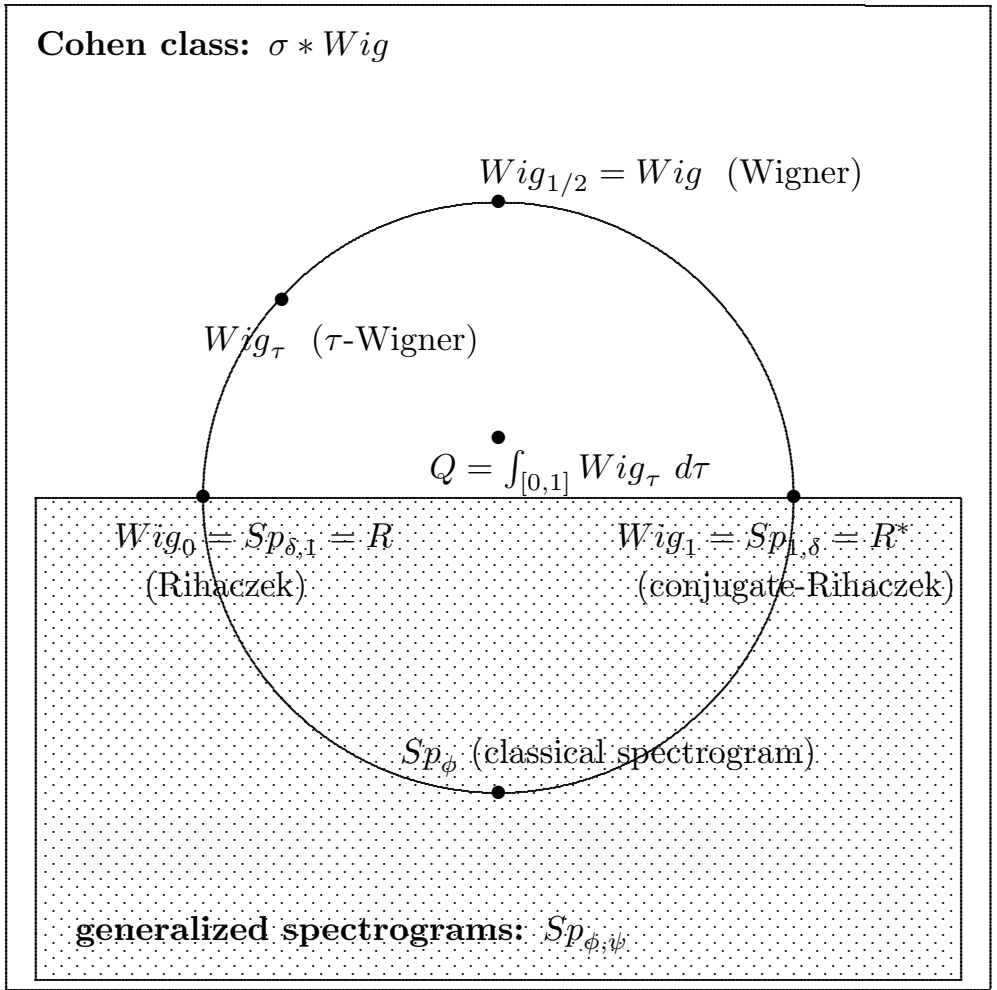

Figure 1. A graphical idea of different types of representations within the Cohen class

So far we have dealt with sesquilinear forms. The existence of a one-to-one association between bounded sesquilinear forms and operators is stated by the following proposition, proved in [1, which permits us to develop a parallel theory for forms and operators.

Proposition 2.4. Let $E, E_{1}$ and $E_{2}$ be Banach spaces, where $E_{2}$ is supposed to be reflexive. 
(a) Let us assume that $\varphi: E_{2}^{*} \times E_{1} \longrightarrow E^{*}$ is a sesquilinear bounded map. Then there exists a unique linear bounded map $a \in E \longrightarrow T_{a} \in B\left(E_{1}, E_{2}\right)$ such that for every $v \in E_{2}^{*}$ we have

$$
\left(v, T_{a} u\right)=\left(\varphi_{v, u}, a\right) .
$$

(b) Conversely, let the application $a \in E \longrightarrow T_{a} \in B\left(E_{1}, E_{2}\right)$ be linear and continuous; then (2.10) defines a sesquilinear bounded map $\varphi: E_{2}^{*} \times E_{1} \rightarrow$ $E^{*}$.

We recall that here duals are considered as spaces of conjugate-linear functionals so that the pairing $(.,$.$) extends the L^{2}$ inner product.

We remark incidentally that this is not the usual association form-operator, as here we have vector-valued forms and we consider quantization instead of single operators.

According to this general property and considering for example the $L^{2}$ space setting, it can be showed that the representations previously mentioned are associated with well-known types of operators. The correspondence is the following:

$$
\begin{array}{llll}
\text { i) } & \text { Generalized spectrogram } & \longleftrightarrow & \text { localization operators; } \\
\text { ii) Rihaczek form } & \longleftrightarrow \text { Kohn - Nirenberg operators; } \\
\text { iii) Conjugate }- \text { Rihaczek form } & \longleftrightarrow \text { operators "with right symbol"; } \\
\text { iv) } \tau-\text { Wigner form } & \longleftrightarrow \tau-\text { Weyl operators } \\
& \text { (in particular Wigner form } & \longleftrightarrow \text { Weyl operators }) .
\end{array}
$$

For completeness we recall the definitions of the classes of operators we have mentioned.

Definition 2.5. Let $a \in \mathcal{S}\left(\mathbb{R}^{2 d}\right)$; then the following classes of operators are defined as a continuous map from $\mathcal{S}\left(\mathbb{R}^{d}\right)$ to itself:

i) Localization operator with symbol $a$ and windows $\phi, \psi \in \mathcal{S}\left(\mathbb{R}^{d}\right)$ :

$$
L_{\phi, \psi}^{a}: f \longrightarrow L_{\phi, \psi}^{a} f(x)=\int_{\mathbb{R}^{2 d}} a(z)\left(f, \phi_{z}\right)_{L^{2}} \psi_{z}(x) d z,
$$

where $z=(y, w) \in \mathbb{R}^{2 d}, \phi_{z}(t)=e^{2 \pi i t \omega} \phi(t-y)$ and analogously for $\psi_{z}$ (see e.g. [23, 14]).

ii) Kohn-Nirenberg pseudo-differential operators:

$$
A^{a}: f \longrightarrow A^{a} f(x)=\int_{\mathbb{R}^{2 d}} e^{2 \pi i(x-y) \omega} a(x, \omega) f(y) d y d \omega
$$

(see e.g. [22, [11]).

iii) Pseudo-differential operators with right symbol $a$ :

$$
B^{a}: f \longrightarrow B^{a} f(x)=\int_{\mathbb{R}^{2 d}} e^{2 \pi i(x-y) \omega} a(y, \omega) f(y) d y d \omega
$$

(see e.g. 16]).

iv) $\tau$-Weyl pseudo-differential operators with $\tau$-symbol $a$ (with $\tau \in[0,1]$ ):

$$
W_{\tau}^{a}: f \longrightarrow W_{\tau}^{a} f(x)=\int_{\mathbb{R}^{2 d}} e^{2 \pi i(x-y) \omega} a((1-\tau) x+\tau y, \omega) f(y) d y d \omega
$$

(see e.g. [16], 11]). 
Of course the latter include Kohn-Nirenberg operators for $\tau=0$, operators with a "right" symbol for $\tau=1$, and Weyl operators:

$$
f \in \mathcal{S}\left(\mathbb{R}^{d}\right) \longrightarrow W^{a} f(x)=\int_{\mathbb{R}^{2 d}} e^{2 \pi i(x-y) \omega} a\left(\frac{x+y}{2}, \omega\right) f(y) d y d \omega \in \mathcal{S}\left(\mathbb{R}^{d}\right)
$$

for $\tau=1 / 2 \quad$ (see e.g. [1], 17, [18, 21]).

We also recall the important formula

$$
L_{\phi, \psi}^{a}=W^{b}, \quad \text { with } b=a * W i g(\psi, \phi),
$$

expressing a localization operator in terms of a Weyl operator, so that all operators considered here are actually pseudo-differential.

Formula (2.10) specializes in each case of (2.11) to a formula connecting a time-frequency representation and a particular class of pseudo-differential operator. More precisely we have the following proposition.

Proposition 2.6. For $f, g \in \mathcal{S}\left(\mathbb{R}^{d}\right), a \in \mathcal{S}\left(\mathbb{R}^{2 d}\right)$ the following equalities hold (with obvious extensions to more general spaces):

$$
\begin{array}{ll}
\text { i) } & \left(g, L_{\phi, \psi}^{a} f\right)_{L^{2}}=\left(\operatorname{Sp}_{\psi, \phi}(g, f), a\right)_{L^{2}}, \\
\text { ii) } & \left(g, A^{a} f\right)_{L^{2}}=(R(g, f), a)_{L^{2}}, \\
\text { iii) } & \left(g, B^{a} f\right)_{L^{2}}=\left(R^{*}(g, f), a\right)_{L^{2}} \\
\text { iv) } & \left(g, W_{\tau}^{a} f\right)_{L^{2}}=\left(W_{i} g_{\tau}(g, f), a\right)_{L^{2}} .
\end{array}
$$

The proof is just a matter of changes to the variables and interchanging order of integrations.

The associations defined by (2.11) and (2.17) clearly constitute the core of the connections between time-frequency analysis and pseudo-differential theory. As far as we know, they were stated more or less implicitly in literature only for the single couples representation-operator without remarking that they are particular cases of a general fact. In section 6 we shall analyze some consequences of these connections, in particular with respect to $L^{p}$ boundedness.

\section{INTEGRAL OF $\tau$-Wigner TRANSFORMS}

This section has a qualitative character and is devoted to the description of the motivations coming from signal analysis which justify the introduction of the new representation $Q=\int_{[0,1]} W_{i} g_{\tau} d \tau$. More precisely, we shall briefly describe the problem of the so-called "ghost" frequencies, which is of paramount importance in applications to signal analysis. Considering for simplicity the one dimensional case, suppose $f$ is a signal with support in the intervals $[a, b]$ and $[c, d](a<b<c<d)$, and consider a time $x$ near the middle point of the interval $[b, c]$, where therefore no frequencies are present in the signal. A very qualitative but enlightening picture of the way the Wigner representation works at the time $x$ is the following (see [6]). The term $f(x+t / 2) \overline{f(x-t / 2)}$ in the Wigner distribution accounts to a "folding" of the past of the signal onto its future; frequencies are then analyzed by taking the Fourier transform. 
For $x$ near the middle of the interval $[b, c]$, as supposed above, the two intervals $[a, b]$ and $[c, d]$ will then overlap and the Wigner transform will show some frequencies at $x$, which in reality do not exist.

Due to the well-known formula

$$
W i g(\hat{f}, \hat{g})(x, \omega)=W i g(f, g)(-\omega, x)
$$

the same phenomenon appears with respect to the frequencies so that, roughly speaking, one can say that the Wigner representation shows a "false" frequency in the middle of any two real frequencies.

These frequencies are called "ghost" or "interference frequencies" and clearly represent a considerable problem in the physical interpretation of the Wigner distribution; see [6] and [7] for details.

Figure 2 shows this phenomenon for a signal of the type we have described (with two frequencies $\omega=2$ and $\omega=4$ appearing in the intervals $[-6,-4]$ and $[3,5]$, respectively); of course, for more complicated signals the "ghost" frequencies will also show much more complicated patterns.

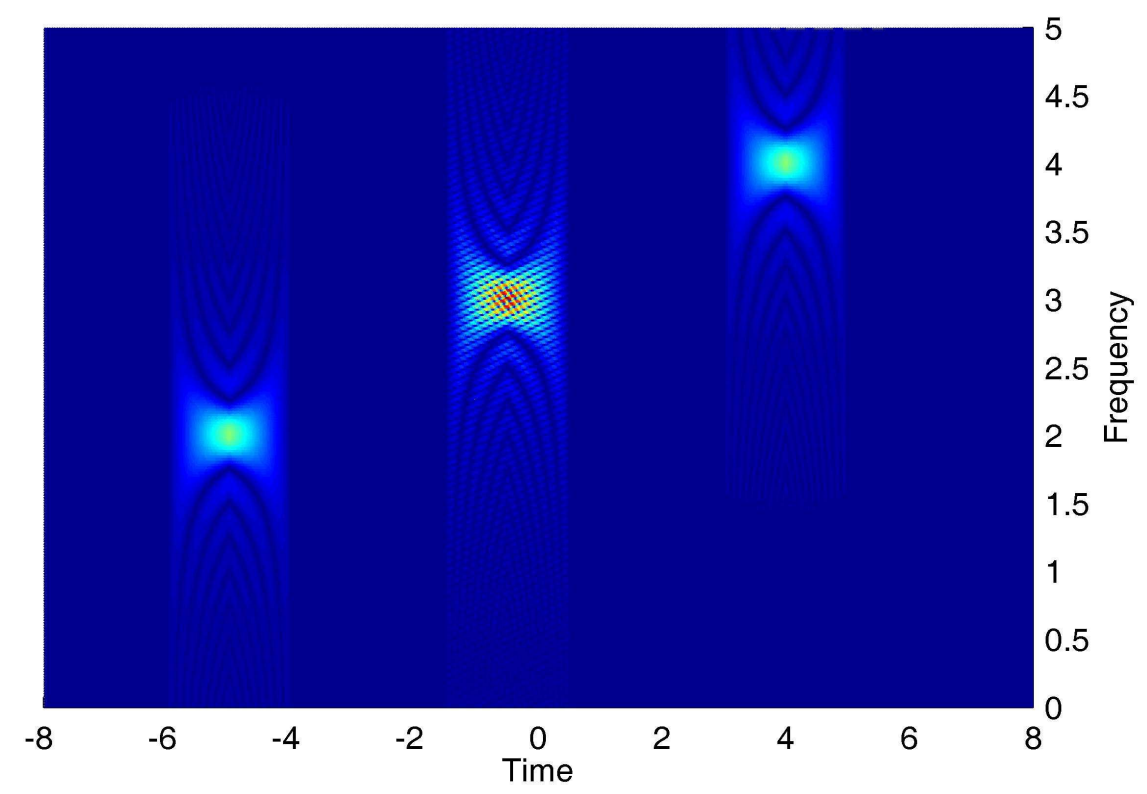

Figure 2. Wig $(f)(x, \omega)$

If we now consider the $W i g_{\tau}$ representations, due to the presence of the parameter, the ghost frequencies are "split" and "shifted" with every $\tau$, whereas, thanks to the support property (Proposition 5.5), the real frequencies appear for all $\tau$ at the "right" place. Figure 3 shows the situation for the $W i g_{\tau}$ with $\tau=0.8$ applied to the same signal as before. 


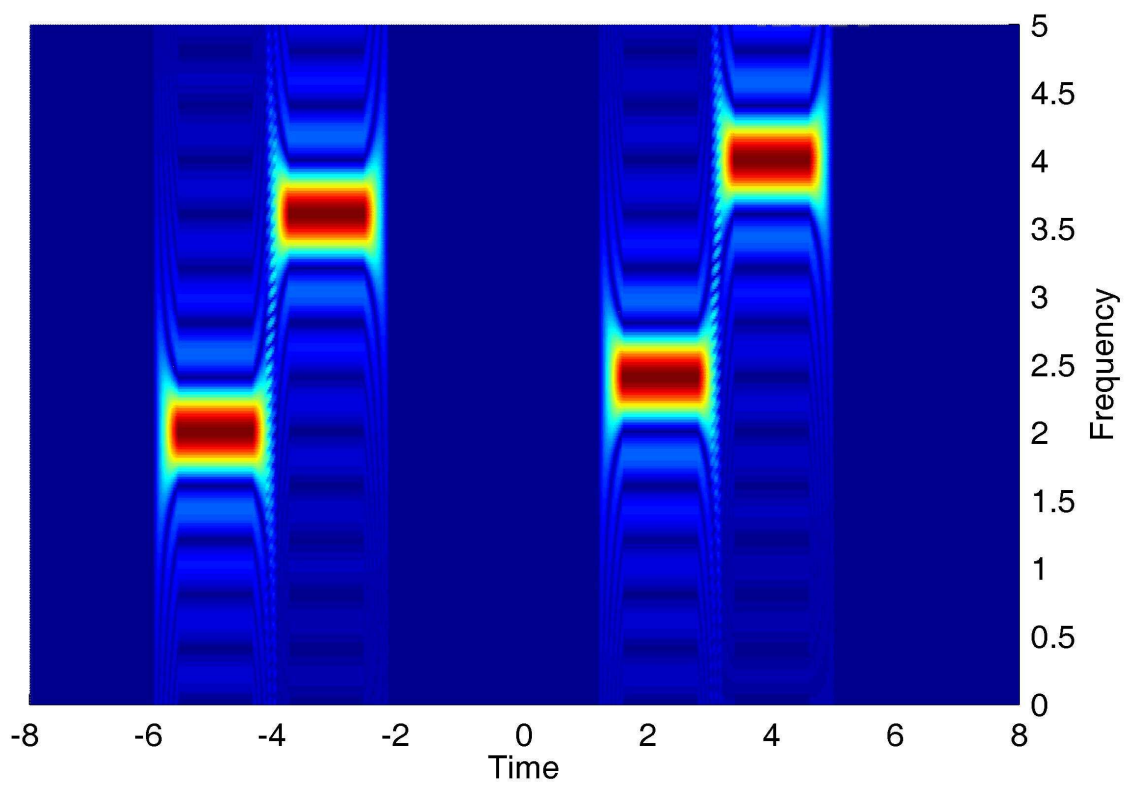

Figure 3. Wig. $_{0.8}(f)(x, \omega)$

We profitably used this fact to construct the form

$$
Q(f)(x, \omega)=\int_{[0,1]} W_{i}(f)(x, \omega) d \tau
$$

which enjoys the same good properties of the Wigner distribution (support property, marginal conditions, etc.) but shows a very reduced presence of "ghost frequencies".

This is actually possible because, in taking the integral over $\tau$, the ghost frequencies of the $W i g_{\tau}$ distributions "spread" on the time-frequency plane and therefore do not "sum", whereas the real frequencies sum one another with every $\tau$. The effect is that the amplitudes of the ghost frequencies will result in being considerably reduced in comparison to the amplitudes of the real frequencies, and therefore the pictures furnished by the representation will be much nearer to physical reality.

Figure 4 shows the result obtained by applying the $Q$ representation to the same signal as in the pictures above: as we can see the ghost frequencies have practically disappeared. (All pictures were obtained with the use of MATLAB using a grid of $800 \times 250$ points and approximating the integrals by a step of 0.01 .)

We also remark that even the real frequencies are better localized in their time intervals, avoiding a certain "blurred margin effect" of the Wigner representation (Figure 2) also due to a question of overlapping supports. 


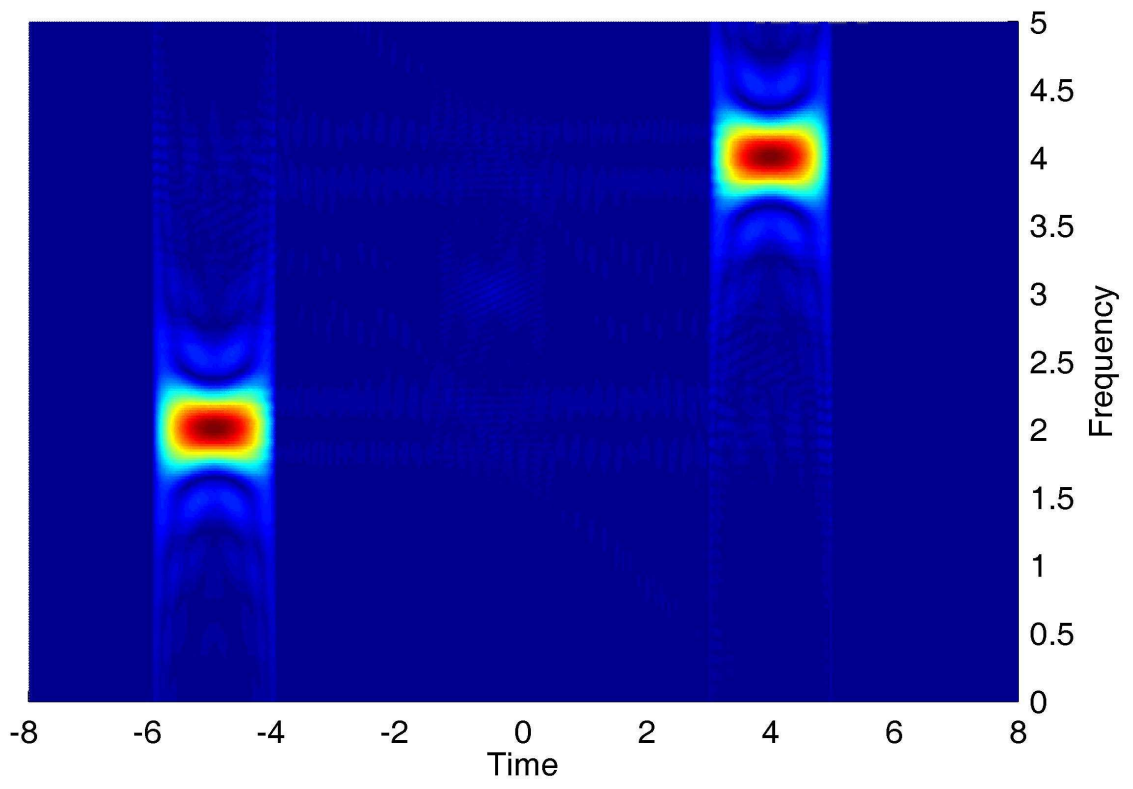

Figure 4. $Q(f)(x, \omega)=\int_{[0,1]} W_{i g_{\tau}}(f)(x, \omega) d \tau$

\section{Precise estimates of interference ReduCtion}

The drastic reduction of the ghost frequencies obtained with the $Q$ representation was shown in the previous section in a pure graphical way. Although the graphical aspect is essential for most applications, it would be desirable to define the problem in mathematical terms and obtain a quantification of this effect in a more precise way.

As far as we know, in the literature this problem has not been put, up to now, in a satisfying and well-defined mathematical form. We propose in this section a possible mathematical formalization and then apply our framework to compare the behavior of the Wigner and the $Q$ representations however, postponing to a further work a deeper study of the subject.

Once a precise concept of interference is defined, the most simple way to proceed would be to fix a single "model" signal containing two frequencies and evaluate their interference with respect to some fixed norm. However such a test would turn out to be extremely dependent on the chosen signal and would have a limited significance.

On the other hand a definition of interference applicable for all signals in $L^{2}\left(\mathbb{R}^{d}\right)$ would present some essential problems out of the aim of this section.

In the following we therefore consider a set of test signals large enough to give general information and for which a simple notion of interference can be defined.

For simplicity we consider the one dimensional case $d=1$. We define as test signals the functions

$$
g_{n}(x)=e^{2 \pi i n x} e^{-\pi x^{2}}
$$


with $x \in \mathbb{R}, n \in \mathbb{Z}$ (i.e. we take modulations $\mu_{n} g_{0}$ of the standard gaussian $\left.g_{0}=e^{-\pi x^{2}}\right)$.

The following proposition guarantees that every signal in $L^{2}(\mathbb{R})$ can be arbitrarily well approximated in the $L^{2}$ norm (at least) on unitary intervals by our test signals, a fact that is interpreted in the sense that the chosen set of test signals is not "too small"; the proof is an easy exercise.

Proposition 4.1. For all $a \in \mathbb{R}$, the set $\left\{g_{n}=\mu_{n} g_{0}: n \in \mathbb{Z}\right\}$ is a base of $L^{2}(a, a+1)$.

For the set of test signals we then define interference as follows.

Definition 4.2. Given a time-frequency representation $\Psi: L^{2}(\mathbb{R}) \times L^{2}(\mathbb{R}) \rightarrow$ $L^{2}\left(\mathbb{R}^{2}\right)$, belonging to the Cohen class, we define as ghost frequency or interference every function of the type

$$
\Psi\left(g_{n}, g_{m}\right)
$$

for $n \neq m$ and $g_{n}, g_{m}$ as in (4.1). The terms $\Psi\left(g_{n}, g_{n}\right)$ are considered, on the other hand, as the representation of the "true" frequency $n$.

One can easily verify in "model cases", such as e.g. the one in the previous section, where true and ghost frequencies defined as above exactly correspond to what is suggested by intuition.

We now need a way to compare the strength by which true frequencies and interferences are represented by $\Psi$. The following lemma will justify our procedure.

Lemma 4.3. Every representation $\Psi$ in the Cohen class satisfies:

i) $\Psi\left(\mu_{\alpha} f, \mu_{\alpha} g\right)(x, \omega)=\Psi(f, g)(x, \omega-\alpha)$,

ii) $\left\|\Psi\left(f, \mu_{\alpha} g\right)\right\|_{L^{2}}=\left\|\Psi\left(f, \mu_{-\alpha} g\right)\right\|_{L^{2}}$,

with $f, g \in L^{2}(\mathbb{R})$ and $\alpha \in \mathbb{R}$.

Proof. The following equality for the Wigner representation is easily verified:

$$
W i g\left(\mu_{\beta} f, \mu_{\alpha} g\right)(x, \omega)=e^{2 \pi i(\beta-\alpha) x} W i g(f, g)\left(x, \omega-\frac{\beta+\alpha}{2}\right) \text {. }
$$

From the fact that the convolution commutes with translations, we obtain part i) of (4.3) for $\alpha=\beta$ and part ii) for $\beta=0$.

From i) of Lemma 4.3 we have

$$
\Psi\left(g_{n}, g_{m}\right)(x, \omega)=\Psi\left(\mu_{n} g_{0}, \mu_{n} \mu_{m-n} g_{0}\right)(x, \omega)=\Psi\left(g_{0}, \mu_{m-n} g_{0}\right)(x, \omega-n)
$$

and then, from ii) of the same lemma, we have that

$$
\left\|\Psi\left(g_{n}, g_{m}\right)\right\|_{L^{2}}=\left\|\Psi\left(g_{0}, \mu_{m-n} g_{0}\right)\right\|_{L^{2}}
$$

only depends on the absolute value $|m-n|$.

A natural measure of the interference is then given by the sequence of the ratios

$$
\alpha_{k}(\Psi)=\frac{\left\|\Psi\left(g_{n}, g_{m}\right)\right\|_{L^{2}}}{\left\|\Psi\left(g_{0}, g_{0}\right)\right\|_{L^{2}}}
$$

with $k=|m-n|$.

For the $W i g_{\tau}$ representations the $\left(\alpha_{k}\right)$ sequence is easily calculated. 
Proposition 4.4. The following holds for the Wig transform:

i) The transform of modulated gaussians is a generalized modulated gaussian; more precisely, for all $n, m \in \mathbb{Z}$, setting $c=2 \tau^{2}-2 \tau+1$, we have

$\operatorname{Wig}_{\tau}\left(g_{n}, g_{m}\right)(x, \omega)$

$=c^{-d / 2} e^{2 \pi i(n-m) x} e^{2 \pi i(\omega-n \tau+(\tau-1) m)(2 \tau-1) x / c} e^{-\pi x^{2} / c} e^{-\pi(\omega-n \tau+(\tau-1) m)^{2} / c}$,

ii) $\left\|W i g_{\tau}\left(g_{n}, g_{m}\right)\right\|_{L^{2}}=2^{-d / 2}$ for every $\tau \in[0,1]$,

iii) $\alpha_{k}\left(W_{i} g_{\tau}\right)=1$ for all $k \in \mathbb{N}$ and $\tau \in[0,1]$.

Proof. Using the technique of completion of squares, a straightforward computation yields the expression of the $\tau$-Wigner in i). The norm evaluation ii) is a direct consequence of i), and $\alpha_{k}=1$ then follows trivially.

As we see, the bad behavior showed with respect to the ghost frequencies is reflected by the fact that $\alpha_{k}\left(W i g_{\tau}\right)$ do not even show a decay as $k$ gets larger. It is interesting to remark that in this case only the typical irregular outline of the ghost frequencies make them recognizable, but their energy (i.e. their $L^{2}$ norm) equals that of the true frequencies. A different situation is shown by the $Q$ representation.

Proposition 4.5. For the $Q$ representation we have:

i) $\left\|Q\left(g_{n}, g_{m}\right)\right\|_{L^{2}}=\left\|\frac{\sin (\pi \eta t)}{\pi \eta t} e^{-\frac{\pi}{2}\left(t^{2}+(\eta-k)^{2}\right)}\right\|_{L^{2}}$, where $k=|n-m|$,

ii) $\alpha_{k}(Q)<1$ and $\lim _{k \rightarrow \infty} \alpha_{k}(Q)=0$.

Proof. i) We have

$$
\begin{aligned}
\left\|Q\left(g_{n}, g_{m}\right)\right\|_{L^{2}} & =\left\|\mathcal{F}\left[\frac{\sin (\pi \eta t)}{\pi \eta t}\right] * W i g\left(g_{n}, g_{m}\right)\right\|_{L^{2}} \\
& =\left\|\frac{\sin (\pi \eta t)}{\pi \eta t} \mathcal{F}^{-1}\left[W i g\left(g_{n}, g_{m}\right)\right](t, \eta)\right\|_{L^{2}},
\end{aligned}
$$

where, as can be directly calculated,

$$
\mathcal{F}^{-1}\left[W i g\left(g_{n}, g_{m}\right)\right](t, \eta)=2^{-d / 2} e^{\pi i(n+m) t} e^{-\frac{\pi}{2}\left(t^{2}+(\eta+n-m)^{2}\right)} .
$$

ii) $\alpha_{k}(Q)<1$ follows immediately from i), and $\lim _{k \rightarrow \infty} \alpha_{k}(Q)=0$ is a consequence of Lebesgue's dominated convergence theorem.

In Figure 5 the rapid decay of the coefficients $\alpha_{k}(Q)$ is shown by a numerical computation of the first 50 terms. 


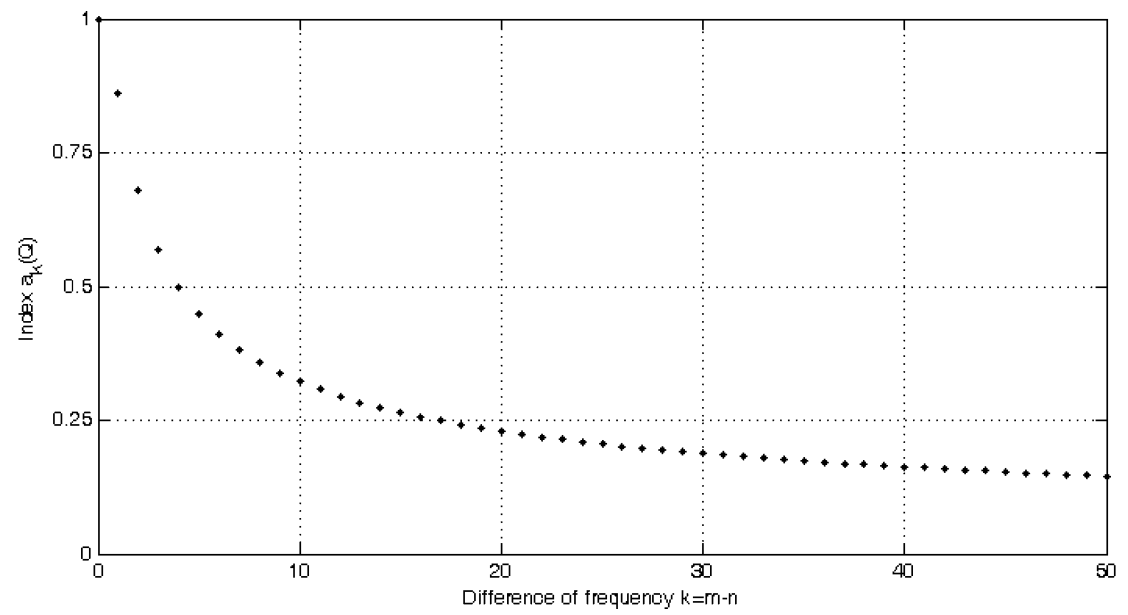

FiguRE $5 . \alpha_{k}(Q)$ for $k \leq 50$

For a given representation $\Psi$, the information given by the sequence $\alpha_{k}(\Psi)$, although detailed, can be easier to handle if it is summarized in a single index. To keep the necessary flexibility we introduce the following definition.

Definition 4.6. Let $\gamma=\left(\gamma_{k}\right)_{k \in \mathbb{N}}, \gamma_{k} \geq 0$, such that $\sum_{k=1}^{\infty} \gamma_{k}=1$. For a representation $\Psi$ in the Cohen class, we define as index $\iota_{\gamma}(\Psi)$ of interference the value

$$
\iota_{\gamma}(\Psi)=\sum_{k=1}^{\infty} \gamma_{k} \alpha_{k}(\Psi)
$$

with $\alpha_{k}(\Psi)$ as in (4.4).

Remark that $\iota_{\gamma}(\Psi)=1$ if, for all $k, \alpha_{k}(\Psi)=1$, and $\iota_{\gamma}(\Psi)=0$ in the ideal case where $\alpha_{k}(\Psi)=0$ for all $k$.

We remark that the dependence of the index $\iota_{\gamma}(\Psi)$ on the sequence $\gamma$ makes it a quite flexible tool. For example, for fixed $N \in \mathbb{N}$, if $\gamma_{k}=1 / N$ for $k \leq N$ and $\gamma_{k}=0$ for $k>N$, then $\iota_{\gamma}(\Psi)$ is the usual average of the interference ratios of the first $N$ differences of frequencies. In this case, whereas obviously $\iota_{\gamma}\left(W i g_{\tau}\right)=1$ for every $\tau \in[0,1]$, we have for the integrated representation that $\iota_{\gamma}(Q)$ is decreasing and infinitesimal when $N$ goes to infinity; for example, in the case $N=50$ described in Figure 5, we get the value $\iota_{\gamma}(Q)=0.2563$.

\section{BASic PRoperties of $\tau$-Wigner AND $Q$ REPRESENTATIONS}

We begin with a justification of Figure 1 by a calculation of the $\tau$-Wigner form in the end points and middle point of the interval $[0,1]$.

Proposition 5.1. For the $\mathrm{Wig}_{\tau}$ representation we have the following three remarkable cases:

a) $W i g_{0}=R=S p_{\delta, 1}$,

b) $W_{i g} / 2=W i g$,

c) $W i g_{1}=R^{*}=S p_{1, \delta}$. 
Proof. a) The equality $\operatorname{Wig}_{0}(f, g)=R(f, g)$ is trivial by putting $\tau=0$ in the expression of $W i g_{\tau}$. We now prove that $R(f, g)=V_{\delta} f(x, \omega) \overline{V_{1} g(x, \omega)}$.

We recall that considering conjugate-linear functionals, the point measure $\delta$ is defined by $(\delta, \varphi)=\overline{\varphi(0)}$, with $\varphi \in \mathcal{S}\left(\mathbb{R}^{d}\right)$. For $\chi \in \mathcal{S}\left(\mathbb{R}^{2 d}\right)$, setting $T_{a} F(t, x)=$ $F(t, t-x)$ and indicating with $\mathcal{F}_{2}$ the partial Fourier transform with respect to the second argument, we have

$$
\begin{aligned}
\left(V_{\delta} f, \chi\right) & =\left(\mathcal{F}_{2} T_{a}(f \otimes \bar{\delta}), \chi\right)=\left(f \otimes \bar{\delta}, T_{a}{ }^{-1} \mathcal{F}_{2}{ }^{-1} \chi\right)=\left(f, \overline{\left(\bar{\delta}, T_{a}{ }^{-1} \mathcal{F}_{2}{ }^{-1} \chi\right)}\right) \\
& =\left(f,\left.\int_{\mathbb{R}^{d}} e^{2 \pi i x \omega} \chi(x-t, \omega) d \omega\right|_{t=0}\right)=\left(f, \int_{\mathbb{R}^{d}} e^{2 \pi i x \omega} \chi(x, \omega) d \omega\right) \\
& =\int_{\mathbb{R}^{2 d}} e^{-2 \pi i x \omega} f(x) \overline{\chi(x, \omega)} d x d \omega=\left(e^{-2 \pi i x \omega} f(x), \chi(x, \omega)\right)
\end{aligned}
$$

for $f \in L^{2}\left(\mathbb{R}^{d}\right)$. Therefore

$$
V_{\delta} f(x, \omega)=e^{-2 \pi i x \omega} f(x) .
$$

Now by relation (5.2) we obtain

$$
V_{1} g(x, \omega)=e^{-2 \pi i x \omega} V_{\hat{1}} \hat{g}(\omega,-x)=e^{-2 \pi i x \omega} V_{\delta} \hat{g}(\omega,-x)=\hat{g}(\omega) .
$$

We conclude that $V_{\delta} f \overline{V_{1} g}(x, \omega)=e^{-2 \pi i x \omega} f(x) \overline{\hat{g}(\omega)}$.

b) is obvious.

c) is analogous to a).

Definition 5.2. A time-frequency representation $\Psi(f)(x, \omega)$ is said to satisfy the Marginal distributions condition if

$$
\int_{\mathbb{R}^{d}} \Psi(f)(x, \omega) d x=|\hat{f}(\omega)|^{2} \text { and } \int_{\mathbb{R}^{d}} \Psi(f)(x, \omega) d \omega=|f(x)|^{2}
$$

for every $f \in L^{2}\left(\mathbb{R}^{d}\right)$.

As $|f(x)|^{2}$ and $|\hat{f}(\omega)|^{2}$ represent the distributions of the energy of $f$ with respect to the time $x$ and the frequencies $\omega$, respectively, they should be the marginal distributions of any ideal time-frequency representation, and therefore the previous definition expresses a quite natural requirement.

Proposition 5.3. The marginal conditions are respected by:

a) the representations $W_{i}(f)$ for every $\tau \in[0,1]$,

b) the integrated representation $Q(f)(x, \omega)=\int_{[0,1]} W i g_{\tau}(f)(x, \omega) d \tau$.

Proof. a) By simple changes of variables we get

$$
\begin{aligned}
\int_{\mathbb{R}^{d}} \operatorname{Wig}_{\tau}(f)(x, \omega) d x & =\int_{\mathbb{R}^{2 d}} e^{-2 \pi i t \omega} f(x+\tau t) \overline{f(x-(1-\tau) t)} d t d x \\
& =\int_{\mathbb{R}^{2 d}} e^{-2 \pi i t \omega} f(y) \overline{f(y-t)} d t d y \\
& =\int_{\mathbb{R}^{2 d}} e^{-2 \pi i y \omega} f(y) \overline{e^{-2 \pi i s \omega} f(s)} d s d y \\
& =|\hat{f}(\omega)|^{2} .
\end{aligned}
$$

In order to prove the other Marginal distribution condition we observe that

$$
W i g_{\tau}(f)(x, \omega)=W i g_{1-\tau}(\hat{f})(\omega,-x)
$$


in fact, we have

$$
\begin{aligned}
W_{g_{1-\tau}(\hat{f})(\omega,-x)} & =\int_{\mathbb{R}^{d}} e^{2 \pi i t x} \hat{f}(\omega+(1-\tau) t) \overline{\hat{f}(\omega-\tau t)} d t \\
& =\int_{\mathbb{R}^{d}} e^{-2 \pi i \omega(y-z)} e^{2 \pi i t(x-y+\tau(y-z))} f(y) \overline{f(z)} d t d y d z \\
& =\int e^{-2 \pi i \omega \tilde{z}} e^{2 \pi i t(x+\tau \tilde{z})} e^{-2 \pi i t y} f(y) \overline{f(y-\tilde{z})} d y d t d \tilde{z} \\
& =\int e^{-2 \pi i \omega \tilde{z}} f(x+\tau \tilde{z}) \overline{f(x-(1-\tau) \tilde{z})} d \tilde{z} \\
& =W i g_{\tau}(f)(x, \omega) .
\end{aligned}
$$

Then, by (5.3) we get

$$
\int_{\mathbb{R}^{d}} W i g_{\tau}(f)(x, \omega) d \omega=\int_{\mathbb{R}^{d}} W i g_{1-\tau}(\hat{f})(\omega,-x) d \omega=|\hat{\hat{f}}(-x)|^{2}=|f(x)|^{2} .
$$

b) The marginal distributions condition for the integrated representation can be immediately deduced from the corresponding properties of the $\mathrm{Wig}_{\tau}$, just by changing the order of integration.

A good time-frequency distribution should not show frequencies where there are none. This can be made precise under different aspects. As we have remarked the "ghost frequencies" are one of these aspects. Another aspect is expressed by the "support" property as follows.

Definition 5.4. Let $H(\operatorname{supp} f)$ be the convex hull of supp $f$ and $H(\operatorname{supp} \hat{f})$ that of supp $\hat{f}$. Let $\Pi_{x}$ and $\Pi_{\omega}$ be the orthogonal projections on the first and the second factors in $\mathbb{R}_{x}^{d} \times \mathbb{R}_{\omega}^{d}$, respectively. A representation $\Psi$ is said to enjoy the "support property" if $\Pi_{x} \operatorname{supp} \Psi(f) \subseteq H(\operatorname{supp} f)$ and $\Pi_{\omega} \operatorname{supp} \Psi(f) \subseteq H(\operatorname{supp} \hat{f})$.

\section{Proposition 5.5.}

a) The representations $W_{i} g_{\tau}$ enjoy the support property for every $\tau \in[0,1]$.

b) The representation $Q$ enjoys the support property.

Proof. a) If $W_{i} g_{\tau}(f)(x, \omega)$ is different from zero, we have by Definition 2.1 that there exists some $t \in \mathbb{R}^{d}$ such that $x+\tau t$ and $x-(1-\tau) t$ belong to supp $f$. Writing $p=x+\tau t$ and $q=x-(1-\tau) t$ we then have that $x=\tau q+(1-\tau) p$ for some $p, q \in \operatorname{supp} f$, and then $x \in H(\operatorname{supp} f)$; this proves that $\Pi_{x} \operatorname{supp} W_{i} g_{\tau}(f) \subset$ $H(\operatorname{supp} f)$. In order to prove that $\Pi_{\omega} \operatorname{supp} W_{i g_{\tau}}(f) \subset H(\operatorname{supp} \hat{f})$ we apply (5.4) and the same arguments as before.

b) By Definition 2.3 if $Q(f)(x, \omega)$ is different from zero, then $W i g_{\tau}(f)(x, \omega)$ must be different from zero for some $\tau$, and then the result follows immediately from the corresponding support properties of the $\tau$-Wigner representations.

We remark that the support property and the absence of "ghost frequencies" are two independent ways of expressing good correspondence between the nature and its representation. For example, all representations in the generalized spectrogram class (with exception of the limit cases of windows $\delta$ and 1) do not have ghost frequencies but do not enjoy the support property; see 2 .

In the next part of this section we will complete the proof of the results concerning $W i g_{\tau}$ and $Q$ contained in Figure 1. In particular we shall prove that $W i g_{\tau}$ belongs 
to the Cohen class for every $\tau$, and it is in the generalized spectrogram only for $\tau=0$ and for $\tau=1$. Moreover, we shall show that the form $Q$ belongs to the Cohen class but not to the generalized spectrogram.

We start by considering $\mathrm{Wig}_{\tau}$.

Proposition 5.6. The form $\mathrm{Wig}_{\tau}(f, g)$ belongs to the Cohen class for every $\tau \in$ $[0,1]$. In particular,

$$
W i g_{\tau}(f, g)(x, \omega)=(\sigma * W i g(f, g))(x, \omega)
$$

for every $f, g \in \mathcal{S}\left(\mathbb{R}^{d}\right)$, where

$$
\sigma= \begin{cases}\frac{2^{d}}{|2 \tau-1|^{d}} e^{2 \pi i \frac{2}{2 \tau-1} x \omega} & \text { for } \tau \neq \frac{1}{2}, \\ \delta & \text { for } \tau=\frac{1}{2}\end{cases}
$$

and $\delta$ is the Dirac distribution.

Proof. The case $\tau=\frac{1}{2}$ is trivial, since by definition $\operatorname{Wig}_{\frac{1}{2}}(f, g)$ coincides with $W i g(f, g)$. Then we have to prove (5.5) for $\tau \neq \frac{1}{2}$. In this case, applying the inverse Fourier transform to (5.5) and taking into account the well-known relation $\mathcal{F}^{-1}(F * G)=\left(\mathcal{F}^{-1} F\right)\left(\mathcal{F}^{-1} G\right)$, we have to prove that

$$
\mathcal{F}^{-1}\left(W_{i} g_{\tau}(f, g)\right)(\xi, t)=\mathcal{F}^{-1} \sigma(\xi, t) \cdot \mathcal{F}^{-1}(W i g(f, g))(\xi, t)
$$

for every $f, g \in \mathcal{S}\left(\mathbb{R}^{d}\right)$. Let us now compute $\mathcal{F}^{-1} \sigma(\xi, t)$. Since $\tau \neq \frac{1}{2}$, by (5.6) we have

$$
\begin{aligned}
\mathcal{F}^{-1} \sigma(\xi, t) & =\frac{2^{d}}{|2 \tau-1|^{d}} \int e^{2 \pi i x \xi} e^{2 \pi i \omega t} e^{2 \pi i \frac{2}{2 \tau-1} x \omega} d x d \omega \\
& =\frac{2^{d}}{|2 \tau-1|^{d}} \int e^{2 \pi i\left(\frac{2}{2 \tau-1} x+t\right)\left(\omega+\frac{2 \tau-1}{2} \xi\right)} e^{-\pi i(2 \tau-1) t \xi} d x d \omega .
\end{aligned}
$$

By the change of variables $\frac{2}{2 \tau-1} x+t=y, \omega+\frac{2 \tau-1}{2} \xi=\rho$ we then get

$$
\mathcal{F}^{-1} \sigma(\xi, t)=e^{-\pi i(2 \tau-1) t \xi} \int e^{2 \pi i y \rho} d y d \rho,
$$

and since $\int e^{2 \pi i y \rho} d y d \rho=1$ we find

$$
\mathcal{F}^{-1} \sigma(\xi, t)=e^{-\pi i(2 \tau-1) t \xi} .
$$

Let us consider the following operators:

$$
T_{s}(F(x, t)):=F\left(x+\frac{t}{2}, x-\frac{t}{2}\right)
$$

and

$$
\tau_{y} G(s):=G(s-y)
$$

we shall also write $\tau_{y}^{[s]}$ to make the shifted variable explicit. By Definition 2.1 we then have

$$
W i g_{\tau}(f, g)(x, \omega)=\mathcal{F}_{t \rightarrow \omega}\left(\tau_{\left(\frac{1}{2}-\tau\right) t}^{[x]}\left(T_{s}(f \otimes \bar{g})\right)\right),
$$

where $f \otimes \bar{g}=f(x) g(t)$ and the translation $\tau$ acts in the $x$-variable. We then define the modulation operator by

$$
\mu_{a} G(\eta):=e^{2 \pi i a \eta} G(\eta)
$$


getting in particular that $\mathcal{F}^{-1}\left(\tau_{a} G(y)\right)=\mu_{a}\left(\mathcal{F}^{-1} G\right)$. As for the translation $\tau$, we shall also write $\mu_{a}^{[\eta]}$ when we need to make the modulated variable $\eta$ explicit.

Now we want to compute the left-hand side of (5.7). Taking into account that the inverse Fourier transform $\mathcal{F}^{-1}$ appearing there is performed with respect to both variables (i.e. it is $\mathcal{F}_{x \rightarrow \xi}^{-1} \mathcal{F}_{\omega \rightarrow t}^{-1}$ ), we have by (5.9)

$$
\begin{aligned}
\mathcal{F}^{-1}\left(\text { Wig }_{\tau}(f, g)\right)(\xi, t) & =\mathcal{F}_{x \rightarrow \xi}^{-1} \mathcal{F}_{\omega \rightarrow t}^{-1} \mathcal{F}_{t \rightarrow \omega}\left(\tau_{\left(\frac{1}{2}-\tau\right) t}^{[x]}\left(T_{s}(f \otimes \bar{g})\right)\right)(\xi, t) \\
& =\mathcal{F}_{x \rightarrow \xi}^{-1}\left(\tau_{\left(\frac{1}{2}-\tau\right) t}^{[x]}\left(T_{s}(f \otimes \bar{g})\right)\right)(\xi, t) \\
& =M_{\left(\frac{1}{2}-\tau\right) t}^{[\xi]}\left(\mathcal{F}_{x \rightarrow \xi}^{-1}\left(T_{s}(f \otimes \bar{g})\right)\right)(\xi, t) \\
& =e^{-\pi i(2 \tau-1) t \xi}\left(\mathcal{F}_{x \rightarrow \xi}^{-1} \mathcal{F}_{\omega \rightarrow t}^{-1}\left(\mathcal{F}_{t \rightarrow \omega} T_{s}(f \otimes \bar{g})\right)\right)(\xi, t) .
\end{aligned}
$$

Since $\mathcal{F}_{t \rightarrow \omega} T_{s}(f \otimes \bar{g})=W i g(f, g)$, by (5.8) the last computations actually show (5.7), and then the proof is complete.

Now we will analyze the relations between the $\tau$-Wigner representation and the generalized spectrogram $S p_{\phi, \psi}(f, g)$; cf. (2.3). As it has already been recalled in Section 2 the set of the sesquilinear forms that can be written as $S p_{\phi, \psi}(f, g)$ for some windows $\phi, \psi$ is a proper subclass of the Cohen class; cf. [2], 3]. We now want to show that the $\tau$-Wigner representation is not in the generalized spectrogram for almost all $\tau$. More precisely, we have the following result.

Proposition 5.7. The $\tau$-Wigner representation $\mathrm{Wig}_{\tau}(\cdot, \cdot)$ belongs to the generalized spectrogram if and only if $\tau=0$ or $\tau=1$.

Proof. The fact that $W i g_{0}$ and $W i g_{1}$ belong to the generalized spectrogram has already been proved in Proposition 5.1. On the other hand, the case $\tau=\frac{1}{2}$ has already been treated in [3, proving that $W i g(f, g)$ cannot be written as $S p_{\phi, \psi}(f, g)$ for any windows $\phi, \psi \in \mathcal{S}^{\prime}\left(\mathbb{R}^{d}\right)$. It then remains to prove that $W i g_{\tau}$ is not in the generalized spectrogram for $0<\tau<1, \tau \neq \frac{1}{2}$. We recall that $S p_{\phi, \psi}(f, g)$ can be expressed as in (2.4). Then, comparing (2.4) with (5.5), we just have to prove that $\sigma$ in (5.6) cannot be expressed as $W i g(\tilde{\psi}, \tilde{\phi})$ for any $\tilde{\phi}, \tilde{\psi} \in \mathcal{S}^{\prime}\left(\mathbb{R}^{d}\right)$. Replacing for simplicity of notation $\tilde{\psi}$ and $\tilde{\phi}$ by $u$ and $v$, since we are considering the case $\tau \neq \frac{1}{2}$, by (5.6) we are reduced to showing that

$$
\operatorname{Wig}(u, v) \neq e^{2 \pi i \frac{2}{2 \tau-1} x \omega}
$$

for every $u, v \in \mathcal{S}^{\prime}\left(\mathbb{R}^{d}\right)$ (we do not care about the constant $\frac{2^{d}}{|2 \tau-1|^{d}}$ in (5.6)). Let us suppose that there exist $u, v \in \mathcal{S}^{\prime}\left(\mathbb{R}^{d}\right)$ such that

$$
W i g(u, v)=e^{2 \pi i \frac{2}{2 \tau-1} x \omega} .
$$

Then, since $\operatorname{Wig}(u, v)=\mathcal{F}_{t \rightarrow \omega} T_{s}(u \otimes \bar{v})$, we have

$$
u \otimes \bar{v}=T_{s}^{-1} \mathcal{F}_{\omega \rightarrow t}^{-1}\left(e^{2 \pi i \frac{2}{2 \tau-1} x \omega}\right) .
$$

Now, $\mathcal{F}_{\omega \rightarrow t}^{-1}\left(e^{2 \pi i \frac{2}{2 \tau-1} x \omega}\right)=\mathcal{F}_{\omega \rightarrow t}^{-1}\left(\mu_{\frac{2}{2 \tau-1} x}^{[\omega]} 1\right)=\tau_{-\frac{2}{2 \tau-1} x}^{[t]}\left(\mathcal{F}_{\omega \rightarrow t}^{-1} 1\right)=\tau_{-\frac{2}{2 \tau-1} x}^{[t]}(\delta(t))$, where we have explicitly written the variable in the Dirac distribution for clearness. We then get

$$
u \otimes \bar{v}=T_{s}^{-1} \tau_{-\frac{2}{2 \tau-1} x}^{[t]}(\delta(t)),
$$


and so, writing $\mathcal{T}:=T_{s}^{-1} \circ \tau_{-\frac{2}{2 \tau-1} x}^{[t]}$, we have

$$
u \otimes \bar{v}=\mathcal{T}(\delta(t)) .
$$

Observe that $\mathcal{T}: \mathcal{S}^{\prime}\left(\mathbb{R}^{d}\right) \rightarrow \mathcal{S}^{\prime}\left(\mathbb{R}^{2 d}\right)$. Explicitly, $\mathcal{T}$ is the extension to distributions of the transformation acting on test functions as $\mathcal{T}(\varphi(t))=\varphi\left(\frac{2-2 \tau}{2 \tau-1} t+\frac{2 \tau}{2 \tau-1} x\right)$ so that if $w \in \mathcal{S}^{\prime}\left(\mathbb{R}^{d}\right), \mathcal{T}(w)$ acts on $\chi \in \mathcal{S}\left(\mathbb{R}^{2 d}\right)$ as

$$
(\mathcal{T}(w), \chi)=\left(1_{x} \otimes w_{t}, c_{\tau} \chi\left(\frac{(2 \tau-1) t-2 \tau x}{2(1-\tau)}, x\right)\right)
$$

with $c_{\tau}=\frac{2 \tau-1}{2-2 \tau}$.

By (5.13) we then have that for every $\phi, \psi \in \mathcal{S}\left(\mathbb{R}^{d}\right)$

$$
\begin{aligned}
(u \otimes \bar{v}, \phi \otimes \psi) & =(\mathcal{T}(\delta), \phi \otimes \psi) \\
& =c_{\tau}\left(1_{x} \otimes \delta_{t}, \psi(x) \phi\left(\frac{(2 \tau-1) t-2 \tau x}{2(1-\tau)}\right)\right) \\
& =c_{\tau} \int \overline{\psi(x)} \overline{\phi\left(\frac{2 \tau x}{2 \tau-2}\right)} d x .
\end{aligned}
$$

Now, $(u \otimes \bar{v}, \phi \otimes \psi)=(u, \phi)(\bar{v}, \psi)$; moreover, we must have $u, v \not \equiv 0$, because otherwise $W i g(u, v)=0$, contradicting (5.12). Then there exists $\phi_{0} \in \mathcal{S}\left(\mathbb{R}^{d}\right)$ such that $\left(u, \phi_{0}\right) \neq 0$. We then have that for every $\psi \in \mathcal{S}\left(\mathbb{R}^{d}\right)$,

$$
(\bar{v}, \psi)=c_{\tau} \int \frac{\overline{\phi_{0}\left(\frac{2 \tau x}{2 \tau-2}\right)}}{\left(u, \phi_{0}\right)} \overline{\psi(x)} d x
$$

and so the distribution $v$ coincides with the function $\bar{c}_{\tau} \overline{\left(u, \phi_{0}\right)}-1 \phi_{0}\left(\frac{2 \tau x}{2 \tau-2}\right)$, in particular $v \in \mathcal{S}\left(\mathbb{R}^{d}\right)$. Similarly, we also get $u \in \mathcal{S}\left(\mathbb{R}^{d}\right)$. By the well-known mapping property $W i g: \mathcal{S}\left(\mathbb{R}^{d}\right) \times \mathcal{S}\left(\mathbb{R}^{d}\right) \rightarrow \mathcal{S}\left(\mathbb{R}^{2 d}\right)$ we then have $W i g(u, v) \in \mathcal{S}\left(\mathbb{R}^{2 d}\right)$, contradicting (5.12). The proof is then complete.

Let us now pass to analyzing the form $Q$; cf. Definition 2.3 .

Proposition 5.8. The form $Q(f, g)(x, \omega)=\int_{[0,1]} W i g_{\tau}(f, g)(x, \omega) d \tau$ belongs to the Cohen class. In particular, for every $f, g \in \mathcal{S}\left(\mathbb{R}^{d}\right)$ we have

$$
Q(f, g)(x, \omega)=(\sigma * W i g(f, g))(x, \omega),
$$

where

$$
\sigma=\mathcal{F}_{\substack{\xi \rightarrow x \\ t \rightarrow \omega}}\left(\frac{\sin (\pi t \xi)}{\pi t \xi}\right) .
$$

Proof. We already know from (5.10) that

$$
\mathcal{F}^{-1}\left(W i g_{\tau}(f, g)\right)(\xi, t)=e^{-\pi i(2 \tau-1) t \xi} \mathcal{F}^{-1}(W i g(f, g))(\xi, t) .
$$

We then have

$$
\begin{aligned}
\mathcal{F}^{-1}(Q(f, g))(\xi, t) & =\left(\int_{0}^{1} e^{-\pi i(2 \tau-1) t \xi} d \tau\right) \mathcal{F}^{-1}(\operatorname{Wig}(f, g))(\xi, t) \\
& =\frac{\sin (\pi t \xi)}{\pi t \xi} \mathcal{F}^{-1}(W i g(f, g))(\xi, t) .
\end{aligned}
$$


The conclusion then follows from the well-known formula $\mathcal{F}(F G)=\mathcal{F}(F) * \mathcal{F}(G)$. Observe that $\frac{\sin (\pi t \xi)}{\pi t \xi} \in L^{\infty}\left(\mathbb{R}^{2 d}\right) \subset \mathcal{S}^{\prime}\left(\mathbb{R}^{2 d}\right)$ so that $\sigma$, cf. (5.15), belongs to $\mathcal{S}^{\prime}\left(\mathbb{R}^{2 d}\right)$.

In order to complete the proof of the results contained in Figure 1 we only have to prove that the form $Q$ does not belong to the generalized spectrogram.

Proposition 5.9. The representation $Q(f, g)$ does not belong to the generalized spectrogram, in the sense that it does not coincide with $S p_{\phi, \psi}(f, g)$ for any choice of $\phi, \psi \in \mathcal{S}^{\prime}\left(\mathbb{R}^{d}\right)$.

Proof. Let us consider for simplicity the case $d=1$. Reasoning as in the proof of Proposition 5.7. comparing the expression of the generalized spectrogram given in (2.4) with the one of $Q$ in (5.14) we just have to prove that there do not exist $u$ and $v$ belonging to $\mathcal{S}^{\prime}(\mathbb{R})$ satisfying

$$
W i g(u, v)=\mathcal{F}_{\substack{\xi \rightarrow x \\ t \rightarrow \omega}}\left(\frac{\sin (\pi t \xi)}{\pi t \xi}\right) .
$$

Let us suppose that such distributions exist. Since $\operatorname{Wig}(u, v)=\mathcal{F}_{t \rightarrow \omega} T_{s}(u \otimes \bar{v})$, applying the inverse Fourier transform we get

$$
T_{s}(u \otimes \bar{v})=\mathcal{F}_{\xi \rightarrow x}\left(\frac{\sin (\pi t \xi)}{\pi t \xi}\right) .
$$

Now, $\frac{\sin (\pi t \xi)}{\pi t \xi}$ can be extended to a bounded continuous function on $\mathbb{R}^{2}$. Then we can perform its partial Fourier transform with respect to the $\xi$ variable, obtaining the fact that $\mathcal{F}_{\xi \rightarrow x}\left(\frac{\sin (\pi t \xi)}{\pi t \xi}\right)$ is a distribution that for $t \neq 0$ coincides with the function

$$
\frac{1}{t} \chi_{\left[-\frac{t}{2}, \frac{t}{2}\right]}(x)
$$

$\chi_{\left[-\frac{t}{2}, \frac{t}{2}\right]}$ being the characteristic function of the interval $\left[-\frac{t}{2}, \frac{t}{2}\right]$. Then by applying $T_{s}^{-1}$ we get that for $X \neq T$ the distribution $u \otimes \bar{v}$ is a function given by

$$
(u \otimes \bar{v})(X, T)=\frac{1}{X-T} \cdot \chi_{\left[-\frac{X-T}{2}, \frac{X-T}{2}\right]}\left(\frac{X+T}{2}\right) .
$$

In particular, in the set $A:=\left\{(X, T) \in \mathbb{R}^{2}: X>0, T<0\right\}$ the distribution $u \otimes \bar{v} \in \mathcal{S}^{\prime}\left(\mathbb{R}^{2}\right)$ coincides with the function $\frac{1}{X-T}$.

Then, since $u \otimes \bar{v} \not \equiv 0$ in $A$, we can fix $\psi_{0} \in C_{0}^{\infty}(\mathbb{R})$ with support in $\mathbb{R}_{-}$in such a way that $\left(\bar{v}, \psi_{0}\right) \neq 0$. We then get that, for every $\phi \in C_{0}^{\infty}(\mathbb{R})$ with support in $\mathbb{R}_{+}$,

$$
(u, \phi)=\int\left\{\frac{1}{\left(\bar{v}, \psi_{0}\right)} \int \frac{1}{X-T} \overline{\psi_{0}(T)} d T\right\} \overline{\phi(X)} d X,
$$

which means that the distribution $u$ coincides with a $C^{\infty}$ function $f_{u}$ in the set $\{X \in \mathbb{R}: X>0\}$. In the same way, we obtain that $v$ coincides with a $C^{\infty}$ function $f_{v}$ in the set $\{T \in \mathbb{R}: T<0\}$; we then have on the set $A$ the factorization

$$
f_{u}(X) \overline{f_{v}(T)}=\frac{1}{X-T} .
$$


We then have for example $f_{u}(X) \overline{v(-2)}=\frac{1}{X+2} \neq 0$ for every $X>0$, and as $f_{u}(X) \neq 0$

$$
\frac{\overline{v(-1)}}{\overline{v(-2)}}=\frac{u(X) \overline{v(-1)}}{u(X) \overline{v(-2)}}=\frac{X+2}{X+1}
$$

for every $X>0$; that is absurd. The proof is then complete.

\section{6. $L^{p}$-BOUNDEDNESS OF FORMS AND PSEUDO-DIFFERENTIAL OPERATORS}

In this last section we study the behavior in the frame of Lebesgue spaces of the $\tau$-Wigner and $Q$ forms, introduced in section 2, also obtaining, in view of Proposition 2.6. results of boundedness for the corresponding $\tau$-Weyl operators $W_{\tau}^{a}$ and their integrals $T_{Q}=\int_{[0,1]} W_{\tau}^{a} d \tau$. More precisely, we begin by observing that we can apply Proposition 2.4 where the operators of Weyl type $W_{\tau}^{a}$ and the $\tau$-Wigner transforms $W i g_{\tau}$ play the role of $T_{a}$ and $\varphi$, respectively. We then have the following result:

Proposition 6.1. In the hypotheses of Proposition 2.4 the following are equivalent for fixed $\tau \in[0,1]$ :

$$
\begin{aligned}
& \text { a) } a \in E \rightarrow W_{\tau}^{a} \in B\left(E_{1}, E_{2}\right) \text { is continuous, } \\
& \text { b) } W i g_{\tau}: E_{2}^{*} \times E_{1} \rightarrow E^{*} \text { is continuous. }
\end{aligned}
$$

We have already introduced in section 2 the Gabor transform

$$
V_{g} f(x, \omega)=\int_{\mathbb{R}^{d}} f(t) \overline{g(t-x)} e^{-2 \pi i t \omega} d t .
$$

In signal processing $g$ is called the window function and $f$ represents the signal. For details and properties see e.g. [6], 10], 13]; a general exposition of this transform in the context of square-integrable representations is presented in 23. Here we just need to recall the following well-known relation between Wigner and Gabor transforms:

$$
W i g(f, g)(x, \omega)=2^{d} e^{4 \pi i x \omega} V_{\tilde{g}} f(2 x, 2 \omega), \tilde{g}(x)=g(-x) .
$$

Later, we shall give a more general version of formula (6.2) involving the $\tau$-Wigner transforms.

For $\tau \in(0,1)$ let us now consider the operator $A_{\tau}$ so defined:

$$
A_{\tau}: h(t) \rightarrow \tilde{h}\left(\frac{1-\tau}{\tau} t\right), \text { with } \tilde{h}(t)=h(-t) .
$$

Then, for $\tau \in(0,1)$ and $h \in L^{p}\left(\mathbb{R}^{d}\right), p \in[1,+\infty]$, we have

$$
\left\|A_{\tau} h\right\|_{L^{p}\left(\mathbb{R}^{d}\right)}=\frac{|\tau|^{\frac{d}{p}}}{|1-\tau|^{\frac{d}{p}}}\|h\|_{L^{p}\left(\mathbb{R}^{d}\right)}
$$

where we mean $\frac{d}{\infty}=0$.

Lemma 6.2. For $\tau \in(0,1)$,

$$
W i g_{\tau}(g, f)(x, \omega)=\frac{1}{|\tau|^{d}} e^{2 \pi i \frac{1}{\tau} x \omega} V_{A_{\tau} f} g\left(\frac{1}{1-\tau} x, \frac{1}{\tau} \omega\right) .
$$


Proof. By the definition of $\tau$-Wigner distribution and by the change of variables $x+\tau t=q$ we obtain

$$
\begin{aligned}
W_{\tau}(g, f)(x, w) & =\int_{\mathbb{R}^{d}} e^{-2 \pi i t \omega} g(x+\tau t) \overline{f(x-(1-\tau) t)} d t \\
& =\frac{1}{|\tau|^{d}} e^{2 \pi i \frac{1}{\tau} x \omega} \int_{\mathbb{R}^{d}} e^{-2 \pi i q \frac{\omega}{\tau}} g(q) \overline{\tilde{f}\left(\frac{1-\tau}{\tau} q-\frac{1}{\tau} x\right)} d q \\
& =\frac{1}{|\tau|^{d}} e^{2 \pi i \frac{1}{\tau} x \omega} \int_{\mathbb{R}^{d}} e^{-2 \pi i q \frac{\omega}{\tau}} g(q) \overline{A_{\tau} f\left(q-\frac{1}{1-\tau} x\right)} d q \\
& =\frac{1}{|\tau|^{d}} e^{2 \pi i \frac{1}{\tau} x \omega} V_{A_{\tau} f} g\left(\frac{1}{1-\tau} x, \frac{1}{\tau} \omega\right) .
\end{aligned}
$$

Let us define $V_{f}^{\tau} g$ as the function

$$
V_{f}^{\tau} g(x, \omega)=V_{f} g\left(\frac{1}{1-\tau} x, \frac{1}{\tau} \omega\right), \quad(x, \omega) \in \mathbb{R}^{2 d} .
$$

Then, by a change of variables, we have, for $\tau \in(0,1)$,

$$
\left\|V_{f}^{\tau} g\right\|_{L^{p}\left(\mathbb{R}^{2 d}\right)}=|\tau|^{\frac{d}{p}}|1-\tau|^{\frac{d}{p}}\left\|V_{f} g\right\|_{L^{p}\left(\mathbb{R}^{2 d}\right)} .
$$

We recall the following result proved in [1].

\section{Proposition 6.3. The Gabor transform}

$$
V:(g, f) \in L^{p^{\prime}}\left(\mathbb{R}^{d}\right) \times L^{p}\left(\mathbb{R}^{d}\right) \rightarrow V_{f} g \in L^{q}\left(\mathbb{R}^{2 d}\right)
$$

is bounded if and only if $q \geq 2$ and $q^{\prime} \leq p \leq q,\left(\frac{1}{q}+\frac{1}{q^{\prime}}=1\right)$. More precisely, in the cases of boundeness we have

$$
\left\|V_{f} g\right\|_{L^{q}} \leq\|g\|_{L^{p^{\prime}}}\|f\|_{L^{p}}
$$

We can now prove that an analogous characterization on Lebesgue spaces holds for the $\tau$-Wigner transform:

Proposition 6.4. Let us fix $q$ and $p$ satisfying $q \geq 2$ and $q^{\prime} \leq p \leq q,\left(\frac{1}{q}+\frac{1}{q^{\prime}}=1\right)$. Then:

i) For $\tau \in(0,1)$, Wig $: L^{p^{\prime}}\left(\mathbb{R}^{d}\right) \times L^{p}\left(\mathbb{R}^{d}\right) \rightarrow L^{q}\left(\mathbb{R}^{2 d}\right)$ is continuous; in particular,

$$
\left\|W i g_{\tau}(g, f)\right\|_{L^{q}} \leq \frac{1}{|1-\tau|^{d\left(\frac{1}{p}-\frac{1}{q}\right)}} \frac{1}{|\tau|^{d\left(1-\frac{1}{p}-\frac{1}{q}\right)}}\|g\|_{L^{p^{\prime}}}\|f\|_{L^{p}}
$$

ii) For $\tau=0, W_{i g}(g, f)(x, \omega)=R(g, f)(x, \omega):=e^{-2 \pi i x \omega} g(x) \bar{f}(\omega)$ and Wig : $L^{q}\left(\mathbb{R}^{d}\right) \times L^{q^{\prime}}\left(\mathbb{R}^{d}\right) \rightarrow L^{q}\left(\mathbb{R}^{2 d}\right)$ is continuous; in particular,

$$
\| \text { Wig } g_{0}(g, f)\left\|_{L^{q}} \leq\right\| g\left\|_{L^{q}}\right\| f \|_{L^{q^{\prime}}} .
$$

iii) For $\tau=1$, Wig $(g, f)(x, \omega)=\overline{R(f, g)}(x, \omega):=e^{2 \pi i x \omega} \overline{f(x)} \hat{g}(\omega)$ and $W i g_{1}$ : $L^{q^{\prime}}\left(\mathbb{R}^{d}\right) \times L^{q}\left(\mathbb{R}^{d}\right) \rightarrow L^{q}\left(\mathbb{R}^{2 d}\right)$ is continuous; in particular,

$$
\left\|W i g_{1}(g, f)\right\|_{L^{q}} \leq\|g\|_{L^{q^{\prime}}}\|f\|_{L^{q}} .
$$

Furthermore, for $p, q$ in the remaining cases the $\tau$-Wigner transform is not bounded. 
Proof. i) From Lemma 6.2, Proposition 6.3 and equations (6.5) and (6.3), we have

$$
\begin{aligned}
\left\|W i g_{\tau}(g, f)\right\|_{L^{q}}^{q} & =\frac{1}{|\tau|^{d q}}\left\|V_{A_{\tau} f}^{\tau} g\right\|_{L^{q}}^{q}=\frac{1}{|\tau|^{d q}}|1-\tau|^{d}|\tau|^{d}\left\|V_{A_{\tau} f} g\right\|_{L^{q}}^{q} \\
& \leq \frac{1}{|\tau|^{d q}}|1-\tau|^{d}|\tau|^{d}\|g\|_{L^{p^{\prime}}}^{q}\left\|A_{\tau} f\right\|_{L^{p}}^{q} \\
& =\frac{1}{|\tau|^{d q}}|1-\tau|^{d}|\tau|^{d} \frac{|\tau|^{\frac{d q}{p}}}{|1-\tau|^{\frac{d q}{p}}}\|g\|_{L^{p^{p}}}^{q}\|f\|_{L^{p}}^{q} \\
& =\frac{1}{|1-\tau|^{d q\left(\frac{1}{p}-\frac{1}{q}\right)}} \frac{1}{|\tau|^{d q\left(1-\frac{1}{p}-\frac{1}{q}\right)}}\|g\|_{L^{p^{\prime}}}^{q}\|f\|_{L^{p}}^{q} .
\end{aligned}
$$

ii) For $\tau=0$, we have

$W i g_{0}(g, f)(x, \omega)=\int_{\mathbb{R}^{d}} e^{-2 \pi i t \omega} g(x) \overline{f(x-t)} d t=e^{-2 \pi i x \omega} g(x) \overline{\hat{f}(\omega)}=R(g, f)(x, \omega)$.

By the Hausdorff-Young inequality we then obtain

$$
\left\|W i g_{0}(g, f)\right\|_{L^{q}}^{q}=\|R(g, f)\|_{L^{q}\left(\mathbb{R}^{2 d}\right)}^{q}=\|g\|_{L^{q\left(\mathbb{R}_{x}^{d}\right)}}^{q}\|\hat{f}\|_{L^{q}\left(\mathbb{R}_{\omega}^{d}\right)}^{q} \leq\|g\|_{L^{q}\left(\mathbb{R}_{x}^{d}\right)}^{q}\|f\|_{L^{q^{\prime}\left(\mathbb{R}_{t}^{d}\right)}}^{q},
$$

which proves ii).

iii) For $\tau=1$ we have, similar to (ii),

$$
\left\|W i g_{1}(g, f)\right\|_{L^{q}}^{q}=\|\overline{R(f, g)}\|_{L^{q}\left(\mathbb{R}^{2 d}\right)}^{q}=\|f\|_{L^{q}\left(\mathbb{R}_{x}^{d}\right)}^{q}\|\hat{g}\|_{L^{q}\left(\mathbb{R}_{\omega}^{d}\right)}^{q} \leq\|f\|_{L^{q}\left(\mathbb{R}_{x}^{d}\right)}^{q}\|g\|_{L^{q^{\prime}\left(\mathbb{R}_{t}^{d}\right)}}^{q},
$$

which proves iii).

Finally, we show the non-boundedness in the remaining cases. Let us define

$$
g(x)=e^{-\pi x^{2}}, \quad g_{\lambda}(x)=e^{-\pi \lambda x^{2}}, \lambda>0, x \in \mathbb{R}^{d} .
$$

Since

$$
\left\|W i g_{\tau}(h, f)\right\|_{L^{q}}^{q}=\frac{|1-\tau|^{d}}{|\tau|^{d(q-1)}}\left\|V A_{\tau} f\right\|_{L^{q}}^{q},
$$

taking $h=g_{\lambda}$ and $f=A_{1-\tau} g$, we obtain that

$$
\frac{\left\|W i g_{\tau}\left(g_{\lambda}, A_{1-\tau} g\right)\right\|_{L^{q}}}{\left\|g_{\lambda}\right\|_{L^{p^{\prime}}}\left\|A_{1-\tau} g\right\|_{L^{p}}}=\frac{1}{|1-\tau|^{d\left(\frac{1}{p}-\frac{1}{q}\right)}} \frac{1}{|\tau|^{d\left(1-\frac{1}{p}-\frac{1}{q}\right)}} \frac{\left\|V_{g} g_{\lambda}\right\|_{L^{q}}}{\|g\|_{L^{p}}\left\|g_{\lambda}\right\|_{L^{p^{\prime}}}},
$$

since $A_{\tau} A_{1-\tau} g=g$.

A direct computation yields

$$
\left(V_{g} g_{\lambda}\right)(x, \xi)=(\lambda+1)^{-\frac{d}{2}} e^{-2 \pi i \frac{1}{\lambda+1} x \xi} g_{\frac{\lambda}{\lambda+1}}(x) g_{\frac{1}{\lambda+1}}(\xi)
$$

and therefore

$$
\left\|V_{g} g_{\lambda}\right\|_{L^{q}}=\frac{1}{(\lambda+1)^{d / 2}}\left\|g_{\frac{\lambda}{\lambda+1}}\right\|_{L_{x}^{q}}\left\|g_{\frac{1}{\lambda+1}}\right\|_{L_{\xi}^{q}}=\frac{1}{(\lambda+1)^{d / 2}}\left(\frac{\lambda q}{\lambda+1}\right)^{-\frac{d}{2 q}}\left(\frac{q}{\lambda+1}\right)^{-\frac{d}{2 q}} .
$$

The result then follows from

$$
\lim _{\lambda \rightarrow 0^{+}} \frac{\left\|V_{g} g_{\lambda}\right\|_{L^{q}}}{\|g\|_{L^{p}}\left\|g_{\lambda}\right\|_{L^{p^{\prime}}}}=+\infty
$$

which holds for $p<q^{\prime}$. 
Remark 6.5. With regard to the cases $\tau=0$ and $\tau=1$, as $W i g_{0}(h, f)=R(h, f)$ and $W_{i} g_{1}=R^{*}(h, f)$, we have that

$$
W i g_{0}: L^{p^{\prime}}\left(\mathbb{R}^{d}\right) \times L^{p}\left(\mathbb{R}^{d}\right) \rightarrow L^{p^{\prime}}\left(\mathbb{R}^{2 d}\right), \quad p \leq 2,
$$

and

$$
W i g_{1}: L^{p^{\prime}}\left(\mathbb{R}^{d}\right) \times L^{p}\left(\mathbb{R}^{d}\right) \rightarrow L^{p}\left(\mathbb{R}^{2 d}\right), \quad p \geq 2,
$$

are the only cases of continuity.

The previous remark then makes precise the non-continuity for $\tau=0$ and $\tau=1$ of $W i g_{0}$ and $W i g_{1}$. We now turn to operators, and we can prove the following:

Theorem 6.6. For $\tau \in[0,1]$ let us consider the operators of Weyl type $W_{\tau}^{a}$ with symbol $a \in L^{q}\left(\mathbb{R}^{2 d}\right)$. Then:

i) If $\tau \in(0,1)$, the quantization

$$
a \in L^{q}\left(\mathbb{R}^{2 d}\right) \rightarrow W_{\tau}^{a} \in B\left(L^{p}\left(\mathbb{R}^{d}\right)\right)
$$

is continuous if and only if $q \leq 2$ and $q \leq p \leq q^{\prime}$, with corresponding norm estimate

$$
\left\|W_{\tau}^{a}\right\|_{B\left(L^{p}\right)} \leq C\|a\|_{L^{q}}, \quad C>0 .
$$

ii) If $\tau=1$, the quantization

$$
a \in L^{q}\left(\mathbb{R}^{2 d}\right) \rightarrow B^{a}=W_{1}^{a} \in B\left(L^{p}\left(\mathbb{R}^{d}\right)\right),
$$

$c f$. (2.14), is continuous if and only if $p=q^{\prime}$ and $q \leq 2$, with corresponding norm estimate

$$
\left\|W_{1}^{a}\right\|_{B\left(L^{q^{\prime}}\right)} \leq C\|a\|_{L^{q},}, \quad C>0 .
$$

iii) If $\tau=0$, the quantization

$$
a \in L^{q}\left(\mathbb{R}^{2 d}\right) \rightarrow A^{a}=W_{0}^{a} \in B\left(L^{p}\left(\mathbb{R}^{d}\right)\right),
$$

cf. (2.13), is continuous if and only if $p=q$ and $q \leq 2$ with corresponding norm estimate

$$
\left\|W_{0}^{a}\right\|_{B\left(L^{q}\right)} \leq C\|a\|_{L^{q}}, \quad C>0 .
$$

Proof. $\quad$ i) Let us suppose at first that $p \neq 1$ and $p \neq \infty$; in this case the result follows from Propositions 6.1 and 6.4 (with $q$ and $q^{\prime}$ interchanged). If $p=1$ and $p=\infty$ we cannot apply Proposition 6.1 , since it requires that $E_{2}$ is reflexive. We then analyze these cases separately, starting by proving the continuity of (6.13). If $p=1$ we must have $q=1$, too. The continuity of $W_{\tau}^{a}: L^{1}\left(\mathbb{R}^{d}\right) \rightarrow L^{1}\left(\mathbb{R}^{d}\right)$ for $a \in L^{1}\left(\mathbb{R}^{2 d}\right)$ can be proved directly by a change of variables in the integration; in particular, we have for $\tau \in(0,1)$ :

$$
\begin{aligned}
\left\|W_{\tau}^{a} u\right\|_{L^{1}\left(\mathbb{R}^{d}\right)} & \leq \int|a((1-\tau) x+\tau y, \omega) u(y)| d y d \omega d x \\
& =\frac{1}{|1-\tau|^{d}} \int_{\mathbb{R}^{d}}\left(\int_{\mathbb{R}^{2 d}}|a(z, \omega)| d z d \omega\right)|u(y)| d y \\
& =\frac{1}{|1-\tau|^{d}}\|a\|_{L^{1}\left(\mathbb{R}^{2 d}\right)}\|u\|_{L^{1}\left(\mathbb{R}^{d}\right)} .
\end{aligned}
$$


Regarding the case $p=\infty$, since we must again have $q=1$, the continuity follows immediately from

$$
\left\|W_{\tau}^{a} u\right\|_{L^{\infty}\left(\mathbb{R}^{d}\right)} \leq \frac{1}{|\tau|^{d}}\|a\|_{L^{1}\left(\mathbb{R}^{2 d}\right)}\|u\|_{L^{\infty}\left(\mathbb{R}^{d}\right)} .
$$

In order to complete the proof of the results in i) we only have to prove the non-continuity of (6.13) when $p=1$ or $p=\infty$. For these values of $p$, let us suppose that the map (6.13) is continuous for some $q \in(1, \infty]$. Then, since it is also continuous for $p=2$ and $q=2$, by interpolation we would also deduce the continuity of (6.13) for some $p \in(1, \infty)$ and $q>\min \left\{p, p^{\prime}\right\}$, where we already know that we do not have boundedness.

ii) For $\tau=1$ and $q \neq 1$, the continuity result follows from Propositions 6.1 and 6.4 (with $q$ and $q^{\prime}$ interchanged), while the non-continuity is a consequence of Proposition 6.1 and Remark 6.5 (with $q$ and $q^{\prime}$ interchanged). In the cases $q=1$ we cannot apply Proposition 6.1, since it requires that $E_{2}$ is reflexive while $L^{q^{\prime}}=L^{\infty}$ is not. The continuity of $W_{1}^{a}: L^{\infty}\left(\mathbb{R}^{d}\right) \rightarrow L^{\infty}\left(\mathbb{R}^{d}\right)$ for $a \in L^{1}\left(\mathbb{R}^{2 d}\right)$ can be proved directly:

$$
\left\|W_{1}^{a} u\right\|_{L^{\infty}\left(\mathbb{R}^{d}\right)} \leq\|a\|_{L^{1}\left(\mathbb{R}^{2 d}\right)}\|u\|_{L^{\infty}\left(\mathbb{R}^{d}\right)} .
$$

The non-continuity in the cases when we cannot apply Proposition 6.1 can be proved as in i).

iii) For $\tau=0$ and $q \neq 1$, the continuity result follows from Propositions 6.1 and 6.4 (with $q$ and $q^{\prime}$ interchanged), while the non-continuity is a consequence of Proposition 6.1 and Remark 6.5 (with $q$ and $q^{\prime}$ interchanged). In the case $q=1$ we cannot apply Proposition 6.1, since it requires that $E_{2}$ is reflexive. The continuity of $W_{0}^{a}: L^{1}\left(\mathbb{R}^{d}\right) \rightarrow L^{1}\left(\mathbb{R}^{d}\right)$ for $a \in L^{1}\left(\mathbb{R}^{2 d}\right)$ can be proved directly:

$$
\begin{aligned}
\left\|W_{0}^{a} u\right\|_{L^{1}\left(\mathbb{R}^{d}\right)} & \leq \int|a(x, \xi) u(y)| d y d \xi d x \\
& =\int_{\mathbb{R}^{d}}\left(\int_{\mathbb{R}^{2 d}}|a(x, \xi)| d x d \xi\right)|u(y)| d y \\
& =\|a\|_{L^{1}\left(\mathbb{R}^{2 d}\right)}\|u\|_{L^{1}\left(\mathbb{R}^{d}\right)} .
\end{aligned}
$$

Also in this case, when we cannot apply Proposition 6.1 we can prove the non-continuity as in i).

Let us remark that, in the previous theorem, the cases of non-continuity of the maps (6.13), 6.14) and (6.15) mean that this map cannot be defined everywhere on $L^{q}\left(\mathbb{R}^{2 d}\right)$; i.e. there exists a symbol $a \in L^{q}\left(\mathbb{R}^{2 d}\right)$ for which $W_{\tau}^{a}$ is not bounded on $L^{p}\left(\mathbb{R}^{d}\right)$. This is actually an immediate consequence of the following general proposition.

Proposition 6.7. The correspondence of operators of Weyl type $a \in L^{q}\left(\mathbb{R}^{2 d}\right) \rightarrow$ $W_{\tau}^{a} \in B\left(L^{p}\left(\mathbb{R}^{d}\right)\right), \tau \in[0,1]$, is continuous if and only if it is everywhere defined.

Proof. Suppose that $a_{j} \rightarrow a$ in $L^{q}\left(\mathbb{R}^{2 d}\right)$ and there exists $A \in B\left(L^{p}\left(\mathbb{R}^{d}\right)\right)$ such that $W_{\tau}^{a_{j}} \rightarrow A$ in $B\left(L^{p}\left(\mathbb{R}^{d}\right)\right)$. Then $a_{j} \rightarrow a$ in $\mathcal{S}^{\prime}\left(\mathbb{R}^{2 d}\right)$ and, therefore, from (2.17), $A=W_{\tau}^{a}$. The graph of the $\tau$-Weyl correspondence is then closed, and the assertion is an immediate consequence of the closed graph theorem. 
Remark 6.8. Theorem 6.6 provides necessary and sufficient conditions for the boundedness of $\tau$-Weyl quantizations, for every $\tau \in[0,1]$. We remark that in the case $\tau=0$ the sufficiency was already proved in [15].

Finally, using the continuity of the map $a \rightarrow W_{\tau}^{a}$ proved in Theorem 6.6 we can show that the bounded operators above are actually compact.

Theorem 6.9. In the same hypotheses as in Theorem 6.6 the operators of Weyl type $W_{\tau}^{a}$ is compact on $L^{p}\left(\mathbb{R}^{d}\right)$.

Proof. Let $a \in L^{q}\left(\mathbb{R}^{d}\right)$ and let us approximate $a$ with a compactly supported function $a_{0}$. Let $\phi \in C_{c}^{\infty}\left(\mathbb{R}^{d}\right)$ and consider the associated approximate identity $\phi_{j}$, $j \in \mathbb{N}$. Then $a_{0} * \phi_{j} \in C_{c}^{\infty}\left(\mathbb{R}^{d}\right)$ so that the kernel of the operator $W_{\tau}^{a_{0} * \phi_{j}}$ is in $\mathcal{S}\left(\mathbb{R}^{d}\right)$. It follows that $W_{\tau}^{a_{0} * \phi_{j}}$ is a regularizing operator on $\mathcal{S}^{\prime}\left(\mathbb{R}^{d}\right)$ and therefore a compact operator on $L^{p}\left(\mathbb{R}^{d}\right)$. As $a_{0} * \phi_{j}$ approximate $a$ in $L^{p}\left(\mathbb{R}^{d}\right)$, from Theorem 6.6 we have that $W_{\tau}^{a}$ is compact.

Let us now consider the integral form of the type

$$
Q=Q(g, f)(x, \omega)=\int_{[0,1]} W_{i}(g, f)(x, \omega) d \tau,
$$

and the corresponding associated operator $T_{Q}^{a}$ having symbol $a$ defined in view of relation (2.10) as usual:

$$
\left(T_{Q}^{a} u, v\right)_{L^{2}\left(\mathbb{R}^{d}\right)}=(a, Q(v, u))_{L^{2}\left(\mathbb{R}^{2 d}\right)} .
$$

We can then state the following result about sufficient conditions for the boundedness of $T_{Q}^{a}$. The interesting point in them is that, on the contrary to what happens with other types of operators, the validity of the boundedness property is dependent on the dimension $d$ of $\mathbb{R}^{d}$. We leave to a further paper the interesting investigation of whether these conditions are a characterization of the boundedness property, and therefore the restrictions in dependence on the dimension are essential or not.

Theorem 6.10. Let us fix $q \leq 2$ and $p$ satisfying

$$
\max \left\{\frac{d p}{d+p}, \frac{d p^{\prime}}{d+p^{\prime}}\right\}<q \leq \min \left\{p, p^{\prime}\right\},
$$

where $p^{\prime}$ is the conjugate of $p$, i.e. $\frac{1}{p}+\frac{1}{p^{\prime}}=1$, and $d$ is the dimension of the space. Then the quantization

$$
a \in L^{q}\left(\mathbb{R}^{2 d}\right) \rightarrow T_{Q}^{a} \in B\left(L^{p}\left(\mathbb{R}^{d}\right)\right)
$$

is continuous, with corresponding norm estimate

$$
\left\|T_{Q}^{a}\right\|_{B\left(L^{p}\right)} \leq C\|a\|_{L^{q}}, \quad C>0 .
$$

Proof. According to Proposition 2.4 where the operator $T_{Q}^{a}$ and the integral form $Q(g, f)$ play the role of $T_{a}$ and $\varphi$, respectively, it follows that the conditions

$$
\begin{aligned}
& \text { i) } a \in E \rightarrow T_{Q}^{a} \in B\left(E_{1}, E_{2}\right) \text { is continuous, } \\
& \text { ii) } Q: E_{2}^{*} \times E_{1} \rightarrow E^{*} \text { is continuous }
\end{aligned}
$$

are equivalent. To obtain our statement it suffices to prove condition ii) when $E^{*}=L^{q^{\prime}}\left(\mathbb{R}^{2 d}\right), q^{\prime} \geq 2, E_{1}=L^{p}\left(\mathbb{R}^{d}\right)$ and $E_{2}^{*}=L^{p^{\prime}}\left(\mathbb{R}^{d}\right)$. Since for hypothesis 
$q^{\prime} \geq \max \left\{p, p^{\prime}\right\}$, from Proposition 6.4 i) it follows that

$$
\begin{aligned}
\left\|\int_{[0,1]} W_{i}(g, f) d \tau\right\|_{L^{q^{\prime}}} & \leq \int_{[0,1]}\left\|W i g_{\tau}(g, f)\right\|_{L^{q^{\prime}}} d \tau \\
& \leq\|g\|_{L^{p^{\prime}}}\|f\|_{L^{p}} \int_{0}^{1} \frac{1}{|1-\tau|^{d\left(\frac{1}{p}-\frac{1}{q^{\prime}}\right)}} \frac{1}{|\tau|^{d\left(1-\frac{1}{p}-\frac{1}{q^{\prime}}\right)}} d \tau .
\end{aligned}
$$

The last integral converges if and only if

$$
\begin{cases}\frac{1}{p}-\frac{1}{q^{\prime}} & <\frac{1}{d}, \\ 1-\frac{1}{p}-\frac{1}{q^{\prime}} & <\frac{1}{d}\end{cases}
$$

that means

$$
\max \left\{\frac{d p}{d+p}, \frac{d p^{\prime}}{d+p^{\prime}}\right\}<q
$$

Remark 6.11. In the case $d=1$ we get $\max \left\{\frac{p}{p+1}, \frac{p^{\prime}}{p^{\prime}+1}\right\}<1$ for every $p$, and we obtain that the integral operator with symbol in $L^{q}$ belongs to $B\left(L^{p}\right)$ for $q \leq p \leq q^{\prime}$.

Remark 6.12. In the case $d=+\infty(d$ tends to $+\infty)$, we have $\max \left\{p, p^{\prime}\right\} \leq q \leq$ $\min \left\{p, p^{\prime}\right\}$, that means $q=2$, so the integral operator with symbol in $L^{2}$ belongs to $B\left(L^{2}\right)$ for every $d$.

\section{REFERENCES}

[1] P. Boggiatto, G. De Donno, A. Oliaro. Weyl quantization of Lebesgue spaces, Math. Nachr., 282, No. 12, 1656-1663 (2009). MR2588828

[2] P. Boggiatto, G. De Donno, A. Oliaro. A class of quadratic time-frequency representations based on the short-time Fourier transform. Operator Theory: Advances and Appl., 172 235249 (2006). MR2308513 (2008e:42020)

[3] P. Boggiatto, G. De Donno, A. Oliaro. Uncertainty principle, positivity and $L^{p}$-boundedness for generalized spectrograms. J. Math. Anal. Appl. 335(1): 93-112 (2007). MR2340308 (2008g:42024)

[4] P. Boggiatto, G. De Donno, A. Oliaro. A unified point of view on time-frequency representations and pseudo-differential operators, Pseudo-differential operators: partial differential equations and time-frequency analysis. Fields Inst. Commun. 52, Amer. Math. Soc., Providence, RI, 2007, 383-399. MR2385338 (2009c:47074)

[5] L. Cohen. Time-Frequency Distributions - A Review. Proc. of IEEE, 77(7), 941-981 (1989).

[6] L. Cohen. Time-Frequency Analysis. Prentice Hall Signal Proc. series, New Jersey, 1995.

[7] L. Cohen. The uncertainty principle for the short-time Fourier transform. Proc. Int. Soc. Opt. Eng. 22563, 80-90 (1995).

[8] G. B. Folland, A. Sitaram. The uncertainty principle: A mathematical survey. J. Fourier Anal. Appl., 3(3), 207-238 (1989). MR.1448337 (98f:42006)

[9] L. Galleani, L. Cohen. The Wigner distribution for classical systems. Physics Letters A, 302, 149-155 (2002). MR1958480 (2004a:81139)

[10] K. Gröchenig. Foundations of Time-Frequency Analysis. Birkhäuser, Boston, 2001. MR1843717 (2002h:42001)

[11] L. Hörmander, The Analysis of Linear Partial Differential Operators, vol. III. SpringerVerlag, Berlin, 1985.

[12] A. J. A. Janssen. Proof of a conjecture on the supports of Wigner distributions. J. Fourier Anal. Appl., 4(6), 723-726 (1998). MR.1666005 (99j:42014)

[13] G. Kaiser. A friendly guide to wavelets. Birkhäuser, Boston, 1994. MR.1287849 (95i:94003)

[14] N. Lerner. The Wick calculus of pseudo-differential operators and some of its applications. Cubo Matematica Educacional, 5 (1), 213-236 (2003). MR.1957713 (2004a:47058) 
[15] A. Mohammed, M.W. Wong, Rihaczek Transform and Pseudo-Differential Operators, Pseudo-differential operators: Partial differential equations and time-frequency analysis, Fields Inst. Commun. 52, Amer. Math. Soc., Providence, RI, 2007, 375-382. MR 2385337 (2009b:47084)

[16] M. A. Shubin. Pseudodifferential operators and spectral theory. Springer-Verlag, Berlin, second edition, 2001. Translated from the 1978 Russian original by Stig I. Andersson. MR1852334 (2002d:47073)

[17] J. Toft, Continuity properties for modulation spaces with applications to pseudo-differential calculus. I. J. Func. An., 207 (2), 399-429 (2004). MR2032995 (2004j:35312)

[18] J. Toft, Continuity properties for modulation spaces with applications to pseudo-differential calculus. II. Ann. Global Anal. Geom., 26, 73-106 (2004). MR2054576 (2005b:47107)

[19] J. Toft, Continuity properties in non-commutative convolution algebras, with applications in pseudo-differential calculus. Bull. Sci. Math. (2), 126, (2002) 115-142. MR 1906240 (2003d:47102)

[20] E. Wigner. On the quantum correction for thermodynamic equilibrium. Phys. Rev. 40, 749759 (1932).

[21] M. W. Wong, Weyl Transforms. Springer-Verlag, 1998. MR.1639461(2000c:47098)

[22] M. W. Wong, An Introduction to Pseudo-Differential Operators. Second Edition. World Sientific, 1999. MR:1698573(2000c:35002)

[23] M. W. Wong. Wavelet Transform and Localization Operators. Birkhäuser-Verlag, Basel, 2002. MR:1918652(2003i:42003)

Dipartimento di Matematica, Università di Torino, Via Carlo Alberto, 10, I-10123 TORINO, ITALY

E-mail address: paolo.boggiatto@unito.it

Dipartimento di Matematica, Università di Torino, Via Carlo Alberto, 10, I-10123 TORINO, ITALY

E-mail address: giuseppe.dedonno@unito.it

Dipartimento di Matematica, Università di Torino, Via Carlo Alberto, 10, I-10123 TORINO, ITALY

E-mail address: alessandro.oliaro@unito.it 